\title{
Minimizing Cost of Assembly of an Interrelated Dimensional Chain Product Using ABC Algorithm
}

\author{
Mahalingam Sivakumar $\left(\mathbb{D},{ }^{1}\right.$ Nagarajan Lenin $\left(\mathbb{D},{ }^{1}\right.$ Kandasamy Jayakrishna $\mathbb{D},^{2}$ \\ and Natarajan Eswara Prasath $\mathbb{D}^{3}$ \\ ${ }^{1}$ Mechanical Engineering, Vel Tech Rangarajan Dr. Sagunthala ReD Institute of Science and Technology, Chennai 600062 , \\ Tamil Nadu, India \\ ${ }^{2}$ Mechanical Engineering, Vellore Institute of Technology, Vellore 632 014, Tamil Nadu, India \\ ${ }^{3}$ Mechanical Engineering, Velammal College of Engineering and Technology, Madurai 625 009, Tamil Nadu, India
}

Correspondence should be addressed to Nagarajan Lenin; n.lenin@gmail.com

Received 29 April 2021; Revised 5 July 2021; Accepted 15 July 2021; Published 30 July 2021

Academic Editor: Hao Gao

Copyright (c) 2021 Mahalingam Sivakumar et al. This is an open access article distributed under the Creative Commons Attribution License, which permits unrestricted use, distribution, and reproduction in any medium, provided the original work is properly cited.

\begin{abstract}
Selective assembly is a method where components made with wider tolerance are grouped into a number of bins. Based on the best combination of the bin, the corresponding group components are randomly selected and matched together to make an assembly. Existing techniques focused on equal group number partitioning of components, equal probability, equal group width, and equal area methods to minimize either clearance variation or surplus parts using different optimization techniques. Mostly, simple assemblies with two or three components are worked by various authors in the literature without considering their original dimension by considering only their component's tolerance. In the present work, components are classified into different unequal group numbers based on their tolerance values. The interrelated dimensional assemblies are made in a single stage by matching the parts based on the best bin combination obtained by the artificial bee colony algorithm. A simple linear assembly and a threearmed knuckle joint assembly are considered examples of problems to demonstrate the effectiveness of the proposed method by minimizing the manufacturing cost.
\end{abstract}

\section{Introduction}

Manufacturing of products involved various manufacturing processes to ensure the products' geometry, shape, size, and accuracy. A part cannot be manufactured precisely to the nominal dimension due to inherent natural variation in men, machine, material, and manufacturing processes. Tolerances of a mechanical assembly have a good bonding with the cost of manufacturing, quality, and reliability of a product. From the view of a designer, tolerance decides the fits, quality, and working of the final assembly. From the manufacturers' perspective, tolerance involves selecting machines, jigs and fixtures, workmanship ability level, machine setup costs, inspection methods, amount of scrap, and rework. Hence, the identification of scientific methods for tolerance allocation to the components of the assembly in view of achieving the manufacturer's objectives is the great motivation to the researchers.

The precise assembly needs close tolerance components to perform the product's required functioning, leading to secondary operations, increasing manufacturing costs. Selective assembly plays a vital role in making precise assemblies from wider tolerance components by classifying and matching parts randomly based on the best bin combinations. The researchers in the different period had used different grouping methods such as equal group number, equal probability, equal group width, and equal area methods to categorize the components into bins. The cost of product/assembly obtained by these methods is mainly based on two factors: clearance variation and surplus parts. As per the existing literature, these methods were contributed to minimizing the manufacturing cost of the product 
with minimum clearance variation and minimum surplus parts. Still, an effective method is required to minimize the manufacturing cost of a product to a further extent by minimizing the clearance variation and keeping zero surplus parts. This alternate method, namely, the unequal grouping method, has been introduced in this work for the stated purpose. This is the novelty and contribution of the proposed work.

Various authors had dealt with the above-said problem environment using nontraditional optimization tools. Important kinds of the literature among them are presented in the next section.

\section{Literature Review}

Precise assembly requires precise components for the smooth functioning of the product. It requires stringent tolerance in the interchangeable assembly, whereas wider tolerance components can make precise assembly in selective assembly. In the literature, most selective assembly techniques focused on either minimizing surplus parts or minimizing the clearance variation in the assembly. Few works on minimizing surplus parts are given below.

The authors handled the surplus parts minimization along with the clearance variation minimization at different periods. Chan and Linn [1] proposed a grouping method for parts of the different distribution with a cumulative distribution function in selective assembly to minimize surplus parts. Thesen and Jantayavichit [2] introduced small buffer storage of parts to evaluate a high-speed station for selective assembly of high-precision automotive components. Kannan and Jayabalan [3] proposed manufacturing mean of the mating parts before production based on the clearance for complex assemblies to minimize surplus parts where the group tolerance was designed to satisfy the clearance specifications of the assembly. Kern [4] developed a closedform equation for various selective assembly techniques to minimize variation and surplus parts with a general approach applicable to different distribution variations. Matsuura and Shinozaki [5] introduced an optimal mean shift to minimize surplus parts for equal width partitioning schemes where the component with smaller variance was manufactured at three shifted means. Few works on minimizing clearance variation are described below.

Various researchers had used different approaches and algorithms to solve the selective assembly problems. A few of them are discussed here. Pugh [6] proposed an assignment algorithm to reduce the variability by alternatively matching small to large partitions using an enhanced BASIC program that generates desired group numbers for the given component distributions. Pugh [7] studied the performance of the selective assembly when a component exhibits dissimilar variances. The author proposed truncating the component distributing more significant variances so that the resulting component variances are equal and discarded the components, which falls at the distribution extremes. Fang and Zhang [8] adopted the concepts of set theory and probability method to develop a predictive model for quantitatively estimating the matchable degree between mating parts for selective assembly. Babu and Asha [9] used Taguchi's loss function to find the difference from the mean values of obtained minimum clearance variation. Very few authors focused on manufacturing cost in selective assembly. It is given below.

The researchers in different time span made the manufacturing cost reduction by solving the selective assembly problems using GA. Kumar and Kannan [10] used GA for solving problems associated with the fan shaft assembly. The appreciable percentage of savings in the manufacturing cost was obtained through GA. Manickam and De [11] proposed a new method to find the combinations of best groups of components for reducing manufacturing cost using GA. Aderiani et al. [12] discussed a multistage approach using GA to solve hole and shaft assembly with the objectives of minimizing the clearance variation and zero surplus parts. Hui et al. [13] used a datadriven modelling approach using the combination of GA and oversampling technique to evaluate the quality of the linear axis assembly of the machine tool. Good results were obtained using the proposed approach than the previous literature.

The authors had used different optimization tools during different periods for solving the selective assembly problems. Authors have used optimization tools such as GA, parallel population GA [14], and simulated annealing [15] in the literature to determine the best bin combination. The artificial bee colony $(\mathrm{ABC})$ algorithm has not yet been implemented in selective assembly techniques. Several applications of the $\mathrm{ABC}$ algorithm in different fields are explained below. Singh [16] addressed an ABC algorithm for the leaf-constrained minimum spanning tree problem and compared the performance of the ABC approach. Computational results showed the superiority of the new $A B C$ approach over all the other approaches. The developed approach obtained better quality solutions in a shorter time. Karaboga and Akay [17] developed a modified ABC algorithm for solving constrained optimization problems. 13 sets of benchmarking problems were considered to evaluate the modified $\mathrm{ABC}$ algorithm, and the developed method outperforms with other methods. The performance of the ABC algorithm also tested for real engineering problems existing in the literature. Karaboga and Ozturk [18] presented a novel clustering approach using the $\mathrm{ABC}$ algorithm. The $\mathrm{ABC}$ algorithm is used to cluster the data on benchmark problems. The performance of the ABC algorithm is compared with the PSO algorithm and the other nine classification techniques from the literature. The simulation results indicated that the $\mathrm{ABC}$ algorithm could efficiently be used for multivariate data clustering.

Asadzadeh [19] proposed a parallel ABC algorithmbased solution methodology to solve job shop scheduling problems. Tian et al. [20] discussed the effective way to identify the disassembly sequence. Tian et al. [21] solved the disassembly problems using the $\mathrm{ABC}$ algorithm to maximize the profit and minimize the energy consumption. Li et al. [22] planned the robot path using an improved $A B C$ algorithm. Chen [23] made few improvements to the existing $\mathrm{ABC}$ algorithm using an escaped foraging strategy. Xu et al. 
[24] proposed an improved multiobjective ABC algorithm to solve multistage resource levelling problems. Furthermore, He et al. [25] proposed a pseudolabel guided ABC algorithm to eliminate the redundant and noise bands from the hyperspectral images. Wang et al. [26] used a multiobjective feature selection algorithm based on the reduction of samples and $\mathrm{ABC}$ algorithm to reduce computation cost. Yong et al. [27] introduced a mixture of different strategies and multiobjective $\mathrm{ABC}$ algorithm to select the unsupervised band of hyperspectral images. Gao et al. [28] proposed three different strategies for the improvement of the $\mathrm{ABC}$ algorithm.

It is concluded from the literature that authors have made significant efforts in minimizing clearance variation and surplus parts using various optimization methods such as GA, parallel population GA, PSO, and simulated annealing. Still, the manufacturers are searching for new methods to reduce the cost of manufacturing to sustain themselves in the global market. In this paper, a new method of grouping concept is introduced to make interrelated dimensional chain product with minimum manufacturing cost without affecting the assembly clearance variation or assembly specification. A concept of different group/bin number for each component according to their tolerance value is introduced. The repetition of group numbers is introduced based on the number of parts available in the particular group. In the existing method, an equal group number is assigned to all components. The best combination of the bin is obtained by implementing the ABC algorithm. The parts of the best bin combination are matched together to form assembly without any surplus parts with less manufacturing cost.

\section{Problem Definition and Methodology}

Tolerance on critical dimension controls the functioning of the product. Stringent tolerance on the functioning dimension creates the necessity of going for a secondary operation, leading to the increased manufacturing cost of the product. Selective assembly converts wider tolerance components into close tolerance assemblies/precision assemblies by matching the classified parts based on the best bin combinations. Two-component assembly with three partitions will have $36(3 ! * 3$ !) combination of bins, whereas five components with six partitions have $(6 ! *$ 6 ! *6!*6!*6!) $1.93492 E+14$ combinations. This makes the situation an NP-hard problem. Reducing group numbers according to the tolerance of the components may reduce the combination of bins to some extent without affecting the functional tolerance. In this work, components are classified into different group numbers rather than the same group number as traditional methods according to their tolerance value. Furthermore, specific group numbers are allowed to repeat based on the number of parts that falls in that particular bin. Hence, the assemblies are made in a single stage. This is the novelty of this work. The ABC algorithm has been adopted to obtain the best bin combinations.
The proposed work has been carried out in two stages; in the first stage, steps $a, b$, and c are carried out. The definitions of symbols used in this manuscript are given in Table 1.

(a) One thousand random dimensions of each component are generated for the known mean and standard deviation. Equation (1) is used to estimate the standard deviation where the tolerance $\pm T_{i}$ is considered:

$$
\sigma_{i}=\frac{T_{i}}{3}
$$

(b) According to tolerance, each component's bin can be decided, and parts are classified, counted, and calculated for each bin's group width using

$$
g w_{i}=\frac{2 T_{i}}{n b_{i}} .
$$

(c) The length of combination of bin and the approximate repetition of bin number of each component are calculated using the equations (3) and (4). The possible bin numbers can be formed as per equation (5):

$$
\begin{gathered}
l b=\max \left(\begin{array}{c}
n c \\
n b_{i} \\
i=1
\end{array}\right)+G * n c, \\
r b_{i j}=\frac{l b * n p_{i j}}{N_{t}}, \\
p b_{i}=\left\{1, \ldots, r b_{i 1} 2, \ldots, r b_{i 2}, \ldots, n b_{i}, \ldots, r b_{i n b_{i}}\right\} .
\end{gathered}
$$

(d) In the second stage, the best bin combinations are determined using the $\mathrm{ABC}$ algorithm. The minimum and maximum clearance values are obtained when mating the components available in the $k^{\text {th }}$ position of the corresponding group using equations (6) and (7), respectively. The clearance variation when mating the components available in the $k^{\text {th }}$ position of the corresponding group is obtained by calculating the difference between the maximum and minimum clearance values. This is expressed in equation (8). The number of assemblies made when mating the components available in the $k^{\text {th }}$ position of the corresponding group is obtained using equation (9). The number of surplus parts of $i^{\text {th }}$ component available is calculated by subtracting the number of assemblies made as per equation (9) from the number of parts of the $i^{\text {th }}$ component in the $k^{\text {th }}$ position group. This is expressed in equation (10). The clearance variation of the assembly is calculated by taking the difference between the maximum and minimum clearance variation. This is explained using equation (11). The total number of assemblies made is calculated by considering the number of assemblies made when mating the components available in all the positions of the corresponding group, and it is determined using equation (12). The combination of bins, $c b_{i k}$, is considered bees where $k$ takes the values from 1 to $l b$. The objective of the 
Table 1: Symbol definition.

\begin{tabular}{|c|c|}
\hline Symbol & Definition \\
\hline$i$ & Component' index; $i=1$ to $n c$ \\
\hline$\mu_{i}$ & Mean value of $i^{\text {th }}$ component \\
\hline$T_{i}$ & Tolerance of $i^{\text {th }}$ component in the proposed work \\
\hline$n b_{i}$ & Number of bins of $i^{\text {th }}$ component \\
\hline$\sigma_{i}$ & Standard deviation of $i^{\text {th }}$ component \\
\hline$d m_{i}$ & Randomly generated dimension of $i^{\text {th }}$ component \\
\hline$n p_{i j}$ & Number of parts of $i^{\text {th }}$ component in $j^{\text {th }}$ bin number \\
\hline$l b$ & Length of bin combination \\
\hline G & Constant varies from 1 to 2 \\
\hline$r p_{i j}$ & Number of repeated $j^{\text {th }}$ bin number for $i^{\text {th }}$ component \\
\hline$p b_{i}$ & Possible bin numbers of $i^{\text {th }}$ component \\
\hline$A_{i}$ & Allocated tolerance of $i^{\text {th }}$ component \\
\hline$M C_{i a}$ & Manufacturing cost of $i^{\text {th }}$ component for allocated tolerance \\
\hline$T_{i \max }$ & Maximum tolerance of $i^{\text {th }}$ component \\
\hline$T_{i \min }$ & Minimum tolerance of $i^{\text {th }}$ component \\
\hline$F 0_{i}, F 1_{i}, F 2_{i}$ & Manufacturing cost model's coefficients of $i^{\text {th }}$ component \\
\hline$M C_{i}$ & Manufacturing cost of $i^{\text {th }}$ component \\
\hline$D_{i \max }$ & Maximum dimension of $i^{\text {th }}$ component \\
\hline$D_{i \min }$ & Minimum dimension of $i^{\text {th }}$ component \\
\hline$A, B, C$ & Dimension of gears $\mathrm{A}, \mathrm{B}$, and $\mathrm{C}$ \\
\hline$X_{1}, X_{2 a}, X_{2 b}, X_{3 a}, X_{3 b}, X_{4}, X_{5}$ & Dimension of KJA with three arms \\
\hline$T_{X 1}, T_{X 2 a}, T_{X 2 b}, T_{X 3 a}, T_{X 3 b}, T_{X 4}, T_{X 5}$ & Tolerance of KJA with three arms \\
\hline$Y_{1}, Y_{2}, Y_{3}$ & Critical dimension \\
\hline$w_{1}, w_{2}, w_{3}$ & Weights for critical dimension \\
\hline SY & Sum of critical dimension after considering weights \\
\hline$T_{a \max }$ & Maximum tolerance of the assembly \\
\hline$T_{a \min }$ & Minimum tolerance of the assembly \\
\hline$C V$ & Clearance variation of the assembly \\
\hline$r b_{i j}$ & Number of repetition of $i^{\text {th }}$ component's $j^{\text {th }}$ group \\
\hline$p b_{i}$ & Possible bin combination of $i^{\text {th }}$ component \\
\hline$N_{j}$ & Number of components available in $j^{\text {th }}$ group of $i^{\text {th }}$ component \\
\hline$N_{t}$ & Total number of parts manufactured for a component \\
\hline$g n_{i}$ & Number of group of $i^{\text {th }}$ component \\
\hline$g w_{i}$ & Group width of $i^{\text {th }}$ component \\
\hline$c b_{i}$ & Randomly generated selective group combination of $i^{\text {th }}$ component \\
\hline$c b_{i k}$ & Group number of $i^{\text {th }}$ component in $k^{\text {th }}$ position of combination of selective groups \\
\hline$C X_{k}$ & Maximum clearance by mating $k^{\text {th }}$ position selective group \\
\hline$C M_{k}$ & Minimum clearance by mating $k^{\text {th }}$ position selective group \\
\hline$C V_{k}$ & Clearance variation obtained by mating $k^{\text {th }}$ position selective groups \\
\hline$N A_{k}$ & Number of assemblies made by mating $k^{\text {th }}$ position selective groups \\
\hline$N_{i k}$ & Number of parts of $i^{\text {th }}$ component in $k^{\text {th }}$ position group \\
\hline$S_{i j}$ & Number of surplus parts available in $j^{\text {th }}$ group of $i^{\text {th }}$ component \\
\hline$C_{a}$ & Clearance variation of the entire assembly \\
\hline$H$ & Total number of assemblies made \\
\hline$n b_{i}$ & Number of bins of $i^{\text {th }}$ component \\
\hline
\end{tabular}

algorithm is to produce assemblies without surplus parts to meet the assembly specification:

$$
\begin{aligned}
C M_{k}= & g w_{i} *\left(c b_{i k}-1\right)+g w_{i+1} * \\
& \left(c b_{i+1 k}-1\right)+\cdots+g w_{n} *\left(c b_{n k}-1\right), \\
C X_{k}= & g w_{i} * c b_{i k}+g w_{i+1} * c b_{i+1 k} \\
& +\cdots+g w_{n} * c b_{n k}, \\
C V_{k}= & C X_{k}-C M_{k}, \\
N A_{k}= & \min \left(N_{i k i=1}^{n}\right),
\end{aligned}
$$

$$
\begin{aligned}
S_{i j} & =N_{i k}-N A_{k}, \\
C_{a} & =\max \left(C X_{k k=1}^{l b}\right)-\min \left(C M_{k k=1}^{l b}\right), \\
H & =\sum_{k=1}^{l b} N A_{k} .
\end{aligned}
$$

\section{ABC Algorithm}

As per the literature survey [29], it is noted that the ABC algorithm is a well-known algorithm due to its simplicity in 
solving optimization problems, high level of flexibility, good robustness, limited control factors, ready to combine with other methods, ease handling of the objective with stochastic nature, quick convergence, and both exploration and exploitation. All these parameters are essential for solving complex problems in selective assembly. Hence, the ABC algorithm is selected in this work.

The intelligent foraging behaviour of artificial honey bees [30] is simulated in the $\mathrm{ABC}$ algorithm. In general, the following types of bees are in any hive: employed, unemployed, and scouts/onlooker bees. The number of employed bees depends on the number of food sources around the hive, where the employed bees are named as scout bees once foods are vanished [31]. In this stage, they start to search for new food sources, described as follows.

(a) All employed bees are assigned food sources in the initial stage.

(b) The search neighbourhood food source by employed bees after reaching the food sources in the memory determines the nectar amount, and according to that, they will dance.

(c) This dance will be identified by unemployed bees within the hive regarding food sources, and they determine the nectar amounts.

(d) Replacement of empty food sources with new food sources by scout bees.

(e) Within steps (a) to (d), the best food source is recorded. The steps starting (b) to (e) are repeated to obtain the top best. Figure 1 shows the flowchart of the $\mathrm{ABC}$ algorithm.

In this work, the combination of bins of all components is considered an artificial bee in the colony. The top out of best food source is considered the total number of assemblies made within the specified assembly specifications. The ABC parameters are assumed based on Nagarajan et al. [32] and are listed in Table 2.

\section{Numerical Illustration}

The effectiveness of the proposed method is demonstrated on both linear assembly and interrelated assembly. Initially, the concept of different group number and approximate repetition of group number based on the probability of parts available in each bin is introduced in linear assembly dealt by Wang et al. [33]. Later, an interrelated dimensional chain product, Knuckle joint assembly with three arms dealt by Sivakumar et al. [34], is considered to reduce the manufacturing cost by implementing the new concept.

5.1. Linear Assembly. A simple gear assembly consists of three gears, A, B, and C, which is considered as an example problem 1 [33]. These gears are assembled linearly in a gear box which is shown in Figure 2.

The dimension of the gears $\mathrm{A}, \mathrm{B}$, and $\mathrm{C}$ is $\varphi 10_{-0.000}^{+0.012}$, $\varphi 12_{-0.000}^{+0.015}$, and $\varphi 14_{-0.000}^{+0.018}$, respectively. If the gears are assembled interchangeably, the dimension of the assembly will be $\varphi 36_{-0.000}^{+0.045}$. A new selective assembly technique used by Wang et al. [33] reduces the assembly clearance variation from 0.045 to 0.0175 . Gears A, B, and C are classified into 4, 5 , and 6 groups, and the dimensional distribution of one thousand random numbers of gears $\mathrm{A}, \mathrm{B}$, and $\mathrm{C}$ is shown in Table 3. The length of bin combination and the approximate repetition of group numbers are calculated using equations (3) and (4). The possible combination of the bin is formed using equation (5) and represented in Table 4 . The best bin combinations obtained by implementing the $\mathrm{ABC}$ algorithm is presented in Table 5. The evaluation details of the best bin combination are given in Table 6. Furthermore, the calculation of the number of assemblies through equations (6)-(12) is explained in Table 6:

$$
\begin{gathered}
l b=\max \left(\begin{array}{c}
n c \\
g n_{i} \\
i=1
\end{array}\right)+G * n c=\max (4,5,6)+1.5 * 6=6+9=15, \\
r b_{i j}=\frac{l b * n p_{i j}}{1000}=r b_{A 1}=\frac{15 * 131}{1000}=1.965=2 .
\end{gathered}
$$

$C M_{1}=3 *(2-1)+3 *(3-1)+3 *(5-1)=21$,

$C X_{1}=3 * 2+3 * 3+3 * 5=30$,

$C V_{1}=30-21=9$,

$N A_{1}=\min (522,483,93)=93$,

$C_{a}=\max (30,24, \ldots, 27,24)$

$-\min (21,15, \ldots, 18,15)=30-15=15$,

$H=93+131+39+12+123+148+93$

$+174+123+31+6+15+12+0+0=1000$.
With reference to the above calculations, the clearance variation $\left(C_{a}=15\right)$ found using the proposed method is less when compared with Wang et al. [33] $\left(C_{a}=17.5\right)$; i.e., $14.3 \%$ reduction in the clearance variation is obtained. Furthermore, the number of surplus parts is zero in the proposed method. Hence, the results showed the effectiveness of the proposed method.

5.2. Interrelated Dimensional Chain Assembly/Product. Knuckle joint assembly with three arms dealt with by Sivakumar et al. [34] has been considered as an example problem in this work. The dimensional details are shown in 


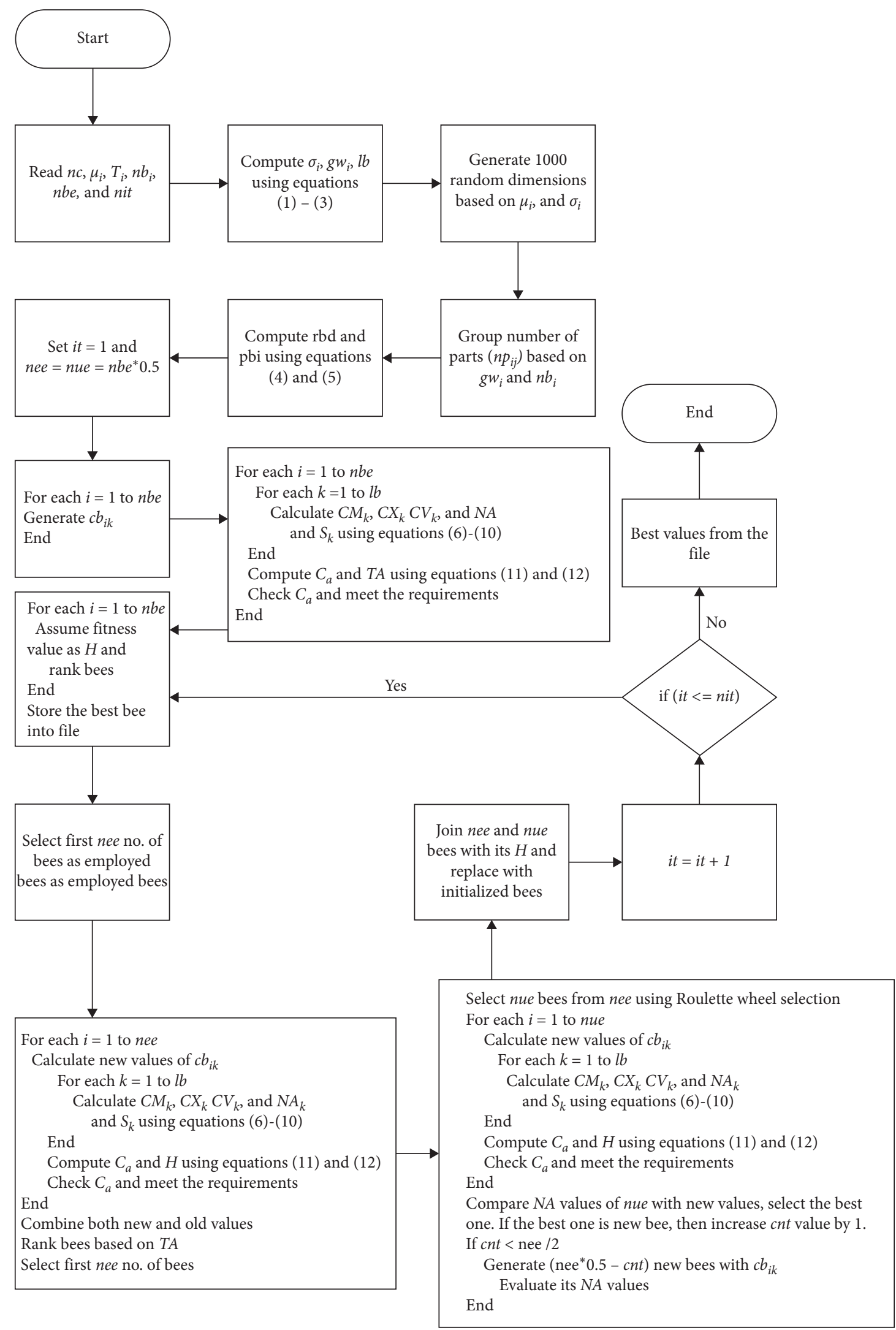

FIgUre 1: Flowchart of the $\mathrm{ABC}$ algorithm. 
TABLE 2: ABC parameters [32].

\begin{tabular}{|c|c|c|}
\hline $\mathrm{ABC}$ parameter & Value/method & Analogy/coding process \\
\hline Bee & A combination of bins of all components & $\begin{array}{c}\text { An assembly with components A, B, and C considering } 3,4 \text {, and } 5 \\
\text { bin partition in which a combination of bins will be considered a } \\
\text { bee. For example, }\{(2,1,3),(4,3,1,2),(3,5,1,2,4)\}\end{array}$ \\
\hline $\begin{array}{l}\text { Total size of bee } \\
\text { colony }\end{array}$ & 40 & 40 different combinations of the bin of components $\mathrm{A}, \mathrm{B}$, and $\mathrm{C}$ \\
\hline $\begin{array}{l}\text { Size of employed } \\
\text { bees }\end{array}$ & $50 \%$ of total bees & $\begin{array}{l}\text { Top } 20 \text { bees based on the ascending order of the clearance variation } \\
\text { of an assembly }\end{array}$ \\
\hline $\begin{array}{l}\text { Size of unemployed } \\
\text { bees }\end{array}$ & $50 \%$ of total bees & The remaining 20 bees out of the total size of bee colony \\
\hline $\begin{array}{l}\text { Selection of food by } \\
\text { onlooker bees }\end{array}$ & Roulette wheel selection method & Selection of best-employed bees based on clearance variation \\
\hline Size of scout bees & $50 \%$ of unemployed bees & Randomly generated ten bees out of 20 unemployed bees \\
\hline Termination criteria & $\begin{array}{l}\text { The specific number of iteration or no change } \\
\text { in the best value for the last } 50 \text { generations is } \\
\text { considered }\end{array}$ & $\begin{array}{l}\text { No change in the clearance variation for the consecutive } 50 \\
\text { iterations }\end{array}$ \\
\hline
\end{tabular}

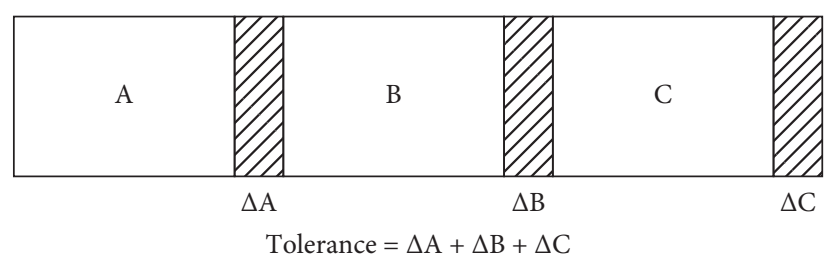

FIgURe 2: Linear gear box assembly.

Table 3: Dimensional distribution of gears A, B, and C for $n b=\left[\begin{array}{lll}4 & 5 & 6\end{array}\right]$.

\begin{tabular}{|c|c|c|c|c|c|c|}
\hline \multirow[t]{2}{*}{ B. no. } & \multicolumn{3}{|c|}{ Number of parts } & \multicolumn{3}{|c|}{$\begin{array}{c}\text { Number of approximate repetition of group } \\
\text { number }\end{array}$} \\
\hline & A & B & $\mathrm{C}$ & $\mathrm{A}$ & $\mathrm{B}$ & $\mathrm{C}$ \\
\hline 1 & 131 & 18 & 12 & 2 & 1 & 1 \\
\hline 2 & 522 & 169 & 123 & 7 & 3 & 2 \\
\hline 3 & 316 & 483 & 411 & 5 & 6 & 5 \\
\hline 4 & 31 & 291 & 355 & 1 & 4 & 5 \\
\hline 5 & & 39 & 93 & & 1 & 1 \\
\hline 6 & & & 6 & & & 1 \\
\hline
\end{tabular}

TABLE 4: The possible combination of the bin $\left(p b_{i}\right)$.

\begin{tabular}{|c|c|c|c|c|c|c|c|c|c|c|c|c|c|c|c|}
\hline C. no. & & & & & & & ssil & $\mathrm{Ac}$ & hat & & & & & & \\
\hline A & 1 & 1 & 2 & 2 & 2 & 2 & 2 & 2 & 2 & 3 & 3 & 3 & 3 & 3 & 4 \\
\hline B & 1 & 2 & 2 & 2 & 3 & 3 & 3 & 3 & 3 & 3 & 4 & 4 & 4 & 4 & 5 \\
\hline C & 1 & 2 & 2 & 3 & 3 & 3 & 3 & 3 & 4 & 4 & 4 & 4 & 4 & 5 & 6 \\
\hline
\end{tabular}

TABLE 5: Best bin combination produced $15 \mu \mathrm{m}$.

\begin{tabular}{|c|c|c|c|c|c|c|c|c|c|c|c|c|c|c|c|}
\hline C. no. & & & & & & & ssib & $\mathrm{nc}$ & nati & & & & & & \\
\hline$\overline{\mathrm{A}}$ & 2 & 1 & 2 & 3 & 3 & 3 & 2 & 2 & 2 & 4 & 3 & 3 & 3 & 3 & 2 \\
\hline B & 3 & 4 & 5 & 4 & 3 & 4 & 3 & 3 & 2 & 2 & 1 & 2 & 1 & 2 & 3 \\
\hline C & 5 & 3 & 3 & 1 & 2 & 3 & 3 & 4 & 4 & 4 & 6 & 4 & 4 & 4 & 3 \\
\hline
\end{tabular}


TABLE 6: Evaluation of best bin combination.

\begin{tabular}{|c|c|c|c|c|c|c|}
\hline \multirow{2}{*}{$k$} & \multicolumn{3}{|c|}{$c b_{k}$} & \multirow{2}{*}{$C M_{k}$} & \multirow{2}{*}{$C X_{k}$} & \multirow{2}{*}{$N A_{k}$} \\
\hline & A & $\mathrm{B}$ & C & & & \\
\hline 1 & 2 & 3 & 5 & 21 & 30 & 93 \\
\hline 2 & 1 & 4 & 3 & 15 & 24 & 131 \\
\hline 3 & 2 & 5 & 3 & 21 & 30 & 39 \\
\hline 4 & 3 & 4 & 1 & 15 & 24 & 12 \\
\hline 5 & 3 & 3 & 2 & 15 & 24 & 123 \\
\hline 6 & 3 & 4 & 3 & 21 & 30 & 148 \\
\hline 7 & 2 & 3 & 3 & 15 & 24 & 93 \\
\hline 8 & 2 & 3 & 4 & 18 & 27 & 174 \\
\hline 9 & 2 & 2 & 4 & 15 & 24 & 123 \\
\hline 10 & 4 & 2 & 4 & 21 & 30 & 31 \\
\hline 11 & 3 & 1 & 6 & 21 & 30 & 6 \\
\hline 12 & 3 & 2 & 4 & 18 & 27 & 15 \\
\hline 13 & 3 & 1 & 4 & 15 & 24 & 12 \\
\hline 14 & 3 & 2 & 4 & 18 & 27 & 0 \\
\hline 15 & 2 & 3 & 3 & 15 & 24 & 0 \\
\hline
\end{tabular}

Figure 3. It consists of seven dimensions and three critical dimensions with interrelated dimensional chains that decide the product's functioning. Table 7 illustrates the allocated tolerance, tolerance cost coefficients, process tolerance limits, and manufacturing cost of each dimension obtained by Sivakumar et al. [34]. The assumed mean dimensions and manufacturing tolerance values of each dimension are represented in Table 8 . The minimum, maximum, and standard deviation of each dimension are calculated using equations (15)-(17). All dimensions and tolerances are in $\mathrm{mm}$ :

$$
\begin{aligned}
D_{i \min } & =\mu_{i}-T_{i}, \\
D_{i \max } & =\mu_{i}+T_{i}, \\
\sigma_{i} & =\frac{T_{i}}{3} .
\end{aligned}
$$

The manufacturing cost of the components is estimated using equation (18) where the cost coefficients are taken from Sivakumar et al. [34]. The interrelated critical dimensions are calculated using equations (19)-(21):

$$
\begin{aligned}
& F_{i}=F 0_{i} * e^{-C 1_{i} t_{i}}+F 2_{i}, \\
& Y_{1}=X_{2 b}-X_{1}, \\
& Y_{2}=X_{3 b}-\left(2 X_{2 a}+X_{2 b}\right), \\
& Y_{3}=X_{4}-\left(2 X_{3 a}+X_{3 b}+X_{5}\right) .
\end{aligned}
$$

The tolerances on critical dimensions are considered the same values as taken by Sivakumar et al. [34] which are given in the following equations:

$$
\begin{aligned}
T_{Y_{1}} & =T_{X_{2 b}}+T_{X 1} \leq 0.2, \\
T_{Y_{2} 1} & =T_{X_{3 b}}+2 T_{X_{2 a}}+T_{X_{2 b}} \leq 0.2,
\end{aligned}
$$

$$
T_{Y_{3}}=T_{X_{4}}+2 T_{X_{3 a}}+T_{X_{3 b}}+T_{X_{5}} \leq 0.2
$$

5.2.1. Implementation of $A B C$ Algorithm. The general structure and the behaviour of bees in the $\mathrm{ABC}$ algorithm are detailed in Section 4. The stepwise procedure of the ABC algorithm is presented in this section.

(a) Population initialization

Generation of a different combination of bins of each component is used for population initialization. A set of combinations of all components of the bin is treated as a bee. For example, a set of possible combinations of bins of the components $\mathrm{A}, \mathrm{B}$, and $\mathrm{C}$ listed in Table 3 is called a bee. This is the way of encoding a bee.

(b) Initialized bees: evaluation

The clearance variation of the assembly is obtained by evaluating the initialized bees and the total number of assemblies made by matching the parts as per the combination of bins. The example calculation is given in Section 5.1.

(c) Identification of search space by employed bees

Equation (25) gives the value of the addition of critical dimensions of the assembly. Based on the increasing order of these values, the bees are listed. The first half count of bees among the list are considered employed bees, and the unemployed bees are the balance:

$$
S Y=w_{1} Y_{1}+w_{2} Y_{2}+w_{3} Y_{3} .
$$

Equation (26) is used to identify the fresh position of the employed bees in the solution space, i.e., the combinations of bins. The number of new bees based on the fresh positions is identical to the number of employed bees. The randomly generated food source $\left(x_{k, j}\right)$ and existing food source 


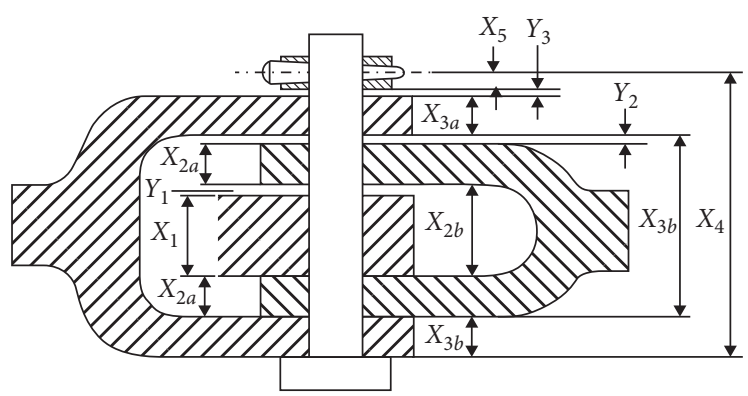

FIGURE 3: KJA with three arms.

TABLE 7: Manufacturing details of KJA with three arms in the existing work [34].

\begin{tabular}{lcccccccc}
\hline C. no. & P. no. & $A_{i}$ & $T_{i \min }$ & $T_{i \max }$ & $F 0_{i}$ & $F 1_{i}$ & $F 2_{i}$ \\
\hline$X_{1}$ & 3 & 0.13762 & 0.01 & 0.15 & 296.40 & 19.5 & 23.82 & 44.07 \\
$X_{2 a}, X_{2 b}$ & 3 & 0.04839 & 0.01 & 0.15 & 296.40 & 19.5 & 23.82 \\
$X_{3 a}, X_{3 b}$ & 3 & 0.04665 & 0.01 & 0.15 & 296.40 & 19.5 & 23.82 \\
$X_{4}$ & 2 & 0.03098 & 0.02 & 0.20 & 82.43 & 16.7 & 21.00 \\
$X_{5}$ & 2 & 0.02513 & 0.01 & 0.10 & 160.43 & 86.7 & 29.20 & 70.14 \\
\hline
\end{tabular}

C. no.: component number; P. no.: process number.

TABLE 8: Manufacturing details of KJA with three arms in the proposed work.

\begin{tabular}{lccccc}
\hline C. no. & $\mu_{i}$ & $\pm T_{i}$ & $D_{\text {imin }}$ & $D_{\text {imax }}$ & $\sigma_{i}$ \\
\hline$X_{1}$ & 25.00 & 0.15 & 24.85 & 25.15 & 0.05 \\
$X_{2 b}$ & 25.50 & 0.15 & 25.35 & 25.65 & 0.05 \\
$X_{2 a}$ & 18.75 & 0.15 & 18.60 & 18.90 & 0.05 \\
$X_{3 b}$ & 63.50 & 0.15 & 63.35 & 63.65 & 0.05 \\
$X_{3 a}$ & 18.75 & 0.15 & 18.60 & 18.90 & 0.05 \\
$X_{4}$ & 107.75 & 0.20 & 107.55 & 107.95 & 0.066667 \\
$X_{5}$ & 6.25 & 0.10 & 6.15 & 6.35 & 0.033333 \\
$Y_{1}=X_{2 b}-X_{1}$ & 0.50 & 0.20 & 0.30 & 0.70 & 23.92 \\
$Y_{2}=X_{3 b}-2 X_{2 a}-X_{2 b}$ & 0.50 & 0.20 & 0.30 & 0.70 & 29.23 \\
$Y_{3}=X_{4}-X_{5}-2 X_{3 a}-X_{3 b}$ & 0.50 & 0.20 & 0.30 & 0.70 & \\
\hline
\end{tabular}

TABLE 9: The best combination of bin, $C V$, and $N A_{k}$ for various bin numbers from 3 to 6 , linear assembly.

\begin{tabular}{|c|c|c|c|c|c|c|c|c|c|c|c|c|c|c|c|c|c|c|c|c|c|c|c|c|c|c|c|c|c|c|c|}
\hline \multirow{2}{*}{$\begin{array}{l}\text { G. no. } \\
3\end{array}$} & \multirow{2}{*}{ C. no. } & \multicolumn{14}{|c|}{ Best combination of bins } & \multirow[t]{2}{*}{$C V$} & \multicolumn{15}{|c|}{ Nos. of assemblies made by matching $k^{\text {th }}$ position bins $\left(N A_{k}\right)$} \\
\hline & & 2 & 1 & 1 & 13 & 33 & 32 & 2 & 2 & 3 & 2 & & & & & & & & & & & & & & & & & & & & \\
\hline 4 & B & 2 & 4 & 2 & 33 & $\begin{array}{ll}3 & 1\end{array}$ & 13 & 3 & 2 & 2 & 3 & 3 & & & & 15.6 & 188 & 84 & 15 & 182 & 16 & 43 & 250 & 73 & 127 & 22 & 0 & 0 & & & \\
\hline 5 & $\mathrm{C}$ & 4 & 3 & 5 & 31 & 13 & 32 & 3 & 3 & 3 & 3 & 3 & & & & & & & & & & & & & & & & & & & \\
\hline 4 & A & 2 & 3 & 1 & 24 & 42 & 22 & 2 & 2 & 3 & 4 & 4 & & & & & & & & & & & & & & & & & & & \\
\hline 5 & B & 4 & 2 & 3 & 53 & 33 & $\begin{array}{ll}3 & 1\end{array}$ & 3 & 3 & 3 & 3 & 1 & & & & 17 & 291 & 169 & 131 & 39 & 16 & 109 & 15 & 19 & 49 & 147 & 12 & 3 & & & \\
\hline 5 & $\mathrm{C}$ & 3 & 4 & 3 & 21 & 13 & 35 & 4 & 2 & 2 & 2 & 2 & & & & & & & & & & & & & & & & & & & \\
\hline 5 & A & 4 & 5 & 2 & 33 & $\begin{array}{ll}3 & 1\end{array}$ & 13 & 2 & 2 & 3 & 3 & 2 & 3 & 3 & 3 & & & & & & & & & & & & & & & & \\
\hline 6 & B & 4 & 3 & 5 & 62 & 23 & 34 & 6 & 4 & 3 & 1 & 3 & 3 & 2 & 1 & 15 & 136 & 11 & 167 & 12 & 93 & 81 & 123 & 11 & 156 & 86 & 6 & 4 & 98 & 14 & 2 \\
\hline 6 & C & 3 & 3 & 3 & 15 & 54 & 42 & 3 & 4 & 3 & 6 & 4 & 4 & 4 & 4 & & & & & & & & & & & & & & & & \\
\hline 4 & A & 1 & 3 & 2 & 34 & 43 & 32 & 3 & 2 & 2 & 2 & 2 & 3 & 3 & 2 & & & & & & & & & & & & & & & & \\
\hline 6 & B & 4 & 4 & 3 & 12 & 24 & 46 & 5 & 5 & 3 & 3 & 2 & 2 & 1 & 3 & 16 & 131 & 123 & 224 & 6 & 31 & 161 & 23 & 12 & 155 & 41 & 15 & 64 & 12 & 2 & 0 \\
\hline 6 & C & 4 & 2 & 4 & 63 & 33 & 33 & 1 & 3 & 3 & 5 & 5 & 5 & 5 & 5 & & & & & & & & & & & & & & & & \\
\hline 4 & A & 2 & 1 & 2 & 33 & 33 & 32 & 2 & 2 & 4 & 3 & 3 & 3 & 3 & 2 & & & & & & & & & & & & & & & & \\
\hline 5 & B & 3 & 4 & 5 & 43 & 34 & 43 & 3 & 2 & 2 & 1 & 2 & 1 & 2 & 3 & 15 & 93 & 131 & 39 & 12 & 123 & 148 & 93 & 174 & 123 & 31 & 6 & 15 & 12 & 0 & 0 \\
\hline 6 & C & 5 & 3 & 3 & 12 & 23 & 33 & 4 & 4 & 4 & 6 & 4 & 4 & 4 & 3 & & & & & & & & & & & & & & & & \\
\hline 5 & A & 2 & 3 & 5 & 42 & 23 & 34 & 1 & 4 & 2 & 2 & 1 & 1 & 3 & 2 & & & & & & & & & & & & & & & & \\
\hline 5 & B & 5 & 2 & 3 & 33 & 34 & 44 & 3 & 3 & 1 & 3 & 4 & 3 & 3 & 2 & 16 & 39 & 169 & 11 & 123 & 186 & 265 & 12 & 6 & 1 & 18 & 95 & 14 & 61 & 0 & 0 \\
\hline 6 & C & 3 & 4 & 3 & 24 & 43 & $\begin{array}{ll}3 & 1\end{array}$ & 6 & 3 & 5 & 3 & 5 & 5 & 4 & 4 & & & & & & & & & & & & & & & & \\
\hline 6 & A & 6 & 4 & 1 & 53 & 33 & 33 & 3 & 3 & 2 & 3 & 2 & 3 & 3 & 3 & & & & & & & & & & & & & & & & \\
\hline 5 & B & 3 & 3 & 4 & 25 & 53 & 33 & 1 & 4 & 4 & 2 & 2 & 2 & 2 & 1 & 17 & 2 & 266 & 62 & 79 & 39 & 123 & 92 & 6 & 12 & 217 & 25 & 2 & 44 & 19 & 12 \\
\hline 6 & C & 3 & 3 & 5 & 33 & 32 & 24 & 6 & 1 & 4 & 3 & 4 & 4 & 5 & 5 & & & & & & & & & & & & & & & & \\
\hline
\end{tabular}


Table 9: Continued.

\begin{tabular}{|c|c|c|c|c|c|c|c|c|c|c|c|c|c|c|c|c|c|c|c|c|c|c|c|c|c|c|c|c|c|c|}
\hline \multirow{2}{*}{$\begin{array}{l}\text { G. no. } \\
6\end{array}$} & \multirow{2}{*}{\multicolumn{2}{|c|}{ C. no. }} & \multicolumn{12}{|c|}{ Best combination of bins } & \multicolumn{2}{|l|}{$C V$} & \multicolumn{14}{|c|}{ Nos. of assemblies made by matching $k^{\text {th }}$ position bins $\left(N A_{k}\right)$} \\
\hline & & 2 & 2 & 4 & 13 & 3 & 1 & 6 & 5 & 33 & 35 & 5 & 2 & 4 & & & & & & & & & & & & & & & & \\
\hline 5 & B & 2 & 3 & 3 & 35 & 4 & 4 & 2 & 4 & 42 & 22 & 1 & 4 & 1 & 17 & 27 & 192 & 266 & 25 & 39 & 200 & 37 & 2 & 41 & 13 & 120 & 20 & 18 & 0 & 0 \\
\hline 4 & $\mathrm{C}$ & 4 & 3 & 2 & 32 & 2 & 3 & 3 & 1 & 33 & 33 & 3 & 2 & 3 & & & & & & & & & & & & & & & & \\
\hline 5 & A & 2 & 3 & 3 & 23 & 3 & 1 & 2 & 2 & 44 & 45 & 4 & 3 & 3 & & & & & & & & & & & & & & & & \\
\hline 6 & 1 & 4 & 4 & 5 & 36 & 3 & 5 & 5 & 3 & 13 & 32 & 2 & 4 & 4 & 16 & 188 & 227 & 16 & 15 & 23 & 168 & 81 & 70 & 65 & 0 & 32 & 11 & 96 & 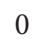 & 0 \\
\hline 5 & ( & 4 & 2 & 1 & 52 & 3 & 3 & 3 & 3 & 33 & 33 & 3 & 3 & 3 & & & & & & & & & & & & & & & & \\
\hline & 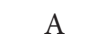 & 4 & 5 & 3 & 23 & 2 & 3 & 1 & 4 & 22 & 25 & 4 & 3 & 3 & & & & & & & & & & & & & & & & \\
\hline 0 & I & 4 & 1 & 4 & 62 & 5 & 3 & 3 & 3 & 32 & 22 & 2 & 3 & 3 & 17 & 41 & 8 & 374 & 23 & 27 & 167 & 33 & 81 & 67 & 99 & 49 & 3 & 28 & 0 & 0 \\
\hline 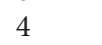 & C & 1 & 2 & 2 & 24 & 3 & 2 & 3 & 2 & 33 & 33 & 3 & 2 & 3 & & & & & & & & & & & & & & & & \\
\hline
\end{tabular}

TABLE 10: The best combination of bin and $C V$ for various bin numbers from 5 to 8 , linear assembly.

\begin{tabular}{|c|c|c|c|c|c|c|c|c|c|c|c|c|c|c|c|c|c|c|c|c|c|c|c|}
\hline G. no. $\left(g n_{i}\right)$ & C. no. & & & & & & & & & est & $\mathrm{om}$ & nat & $n$ & bir & & & & & & & & & $C V$ \\
\hline 5 & $\mathrm{~A}$ & 4 & 2 & 3 & 1 & 3 & 3 & 3 & 2 & 3 & 3 & 2 & 4 & 2 & 5 & 4 & 4 & 3 & 3 & & & & \\
\hline 6 & B & 2 & 5 & 4 & 4 & 4 & 4 & 1 & 3 & 6 & 3 & 6 & 3 & 3 & 3 & 3 & 1 & 5 & 3 & & & & 15.21 \\
\hline 7 & $\mathrm{C}$ & 5 & 4 & 3 & 4 & 2 & 4 & 7 & 6 & 1 & 5 & 4 & 5 & 4 & 4 & 4 & 4 & 4 & 4 & & & & \\
\hline 5 & A & 3 & 2 & 5 & 2 & 2 & 2 & 1 & 3 & 4 & 4 & 3 & 3 & 3 & 4 & 4 & 4 & 4 & 3 & 2 & 1 & 3 & \\
\hline 6 & B & 3 & 5 & 4 & 4 & 6 & 4 & 4 & 4 & 4 & 4 & 4 & 2 & 2 & 1 & 2 & 2 & 2 & 2 & 3 & 4 & 4 & 16.45 \\
\hline 8 & $\mathrm{C}$ & 4 & 5 & 3 & 6 & 3 & 4 & 5 & 5 & 1 & 2 & 3 & 8 & 7 & 4 & 4 & 7 & 3 & 7 & 5 & 8 & 5 & \\
\hline
\end{tabular}

TABLE 11: Assembly specifications for various bin numbers, KJA with three arms.

\begin{tabular}{|c|c|c|c|c|c|c|c|}
\hline Pr. no. & Bin number $(n b)$ & $C$ & $l b$ & $Y_{1}$ & $Y_{2}$ & $Y_{3}$ & $H$ \\
\hline \multirow{2}{*}{1} & \multirow{5}{*}[\begin{array}{lllllll}{3}&{3}&{3}&{3}&{3}&{4}&{3}\end{array}]{} & \multirow{2}{*}{1} & \multirow{2}{*}{17} & 0.4 & 0.6 & 0.6333333 & 1000 \\
\hline & & & & 0.5 & 0.6 & 0.6666667 & 1000 \\
\hline \multirow{3}{*}{2} & & \multirow{3}{*}{1.5} & \multirow{3}{*}{21} & 0.4 & 0.5 & 0.6666667 & 1000 \\
\hline & & & & 0.6 & 0.6 & 0.6666667 & 1000 \\
\hline & & & & 0.5 & 0.6 & 0.6666667 & 1000 \\
\hline \multirow{5}{*}{3} & & \multirow{5}{*}{1.5} & \multirow{5}{*}{24} & 0.525 & 0.525 & 0.6766667 & 1000 \\
\hline & & & & 0.375 & 0.6 & 0.6733333 & 1000 \\
\hline & & & & 0.6 & 0.6 & 0.695 & 1000 \\
\hline & & & & 0.45 & 0.525 & 0.6166667 & 1000 \\
\hline & {$\left[\begin{array}{lllllll}4 & 4 & 4 & 4 & 4 & 5 & 3\end{array}\right]$} & & & 0.6 & 0.675 & 0.6816667 & 1000 \\
\hline \multirow{3}{*}{4} & & \multirow{3}{*}{1.8} & \multirow{3}{*}{26} & 0.525 & 0.6 & 0.6866667 & 1000 \\
\hline & & & & 0.45 & 0.675 & 0.6766667 & 1000 \\
\hline & & & & 0.375 & 0.525 & 0.6816667 & 1000 \\
\hline \multirow{3}{*}{5} & \multirow{3}{*}[\begin{array}{lllllll}{5}&{4}&{5}&{4}&{4}&{4}&{5}\end{array}]{} & \multirow{3}{*}{1.5} & \multirow{3}{*}{25} & 0.54 & 0.69 & 0.66 & 1000 \\
\hline & & & & 0.48 & 0.555 & 0.68 & 1000 \\
\hline & & & & 0.42 & 0.57 & 0.655 & 1000 \\
\hline \multirow{3}{*}{6} & \multirow{3}{*}[\begin{array}{lllllll}{4}&{4}&{4}&{4}&{4}&{4}&{6}\end{array}]{} & \multirow{3}{*}{1.5} & \multirow{3}{*}{26} & 0.375 & 0.525 & 0.675 & 1000 \\
\hline & & & & 0.525 & 0.525 & 0.6083333 & 1000 \\
\hline & & & & 0.45 & 0.675 & 0.6 & 1000 \\
\hline \multirow{4}{*}{7} & \multirow{4}{*}[\begin{array}{lllllll}{5}&{5}&{4}&{4}&{4}&{4}&{6}\end{array}]{} & \multirow{4}{*}{1.5} & \multirow{4}{*}{26} & 0.48 & 0.495 & 0.6916667 & 1000 \\
\hline & & & & 0.42 & 0.555 & 0.625 & 1000 \\
\hline & & & & 0.465 & 0.6 & 0.6333333 & 1000 \\
\hline & & & & 0.465 & 0.675 & 0.6083333 & 1000 \\
\hline \multirow{3}{*}{8} & \multirow{3}{*}[\begin{array}{lllllll}{5}&{4}&{5}&{4}&{4}&{4}&{6}\end{array}]{} & \multirow{3}{*}{1.5} & \multirow{3}{*}{27} & 0.36 & 0.6 & 0.6666667 & 1000 \\
\hline & & & & 0.48 & 0.555 & 0.6583333 & 1000 \\
\hline & & & & 0.42 & 0.66 & 0.6583333 & 1000 \\
\hline \multirow{3}{*}{9} & & & & 0.42 & 0.51 & 0.6633333 & 1000 \\
\hline & {$\left[\begin{array}{lllllll}5 & 4 & 5 & 4 & 5 & 4 & 6\end{array}\right]$} & 1.5 & 27 & 0.48 & 0.525 & 0.6716667 & 1000 \\
\hline & & & & 0.48 & 0.48 & 0.6516667 & 1000 \\
\hline
\end{tabular}


TABle 11: Continued.

\begin{tabular}{|c|c|c|c|c|c|c|c|}
\hline Pr. no. & Bin number $(n b)$ & $C$ & $l b$ & $Y_{1}$ & $Y_{2}$ & $Y_{3}$ & $H$ \\
\hline 10 & \multirow{10}{*}[\begin{array}{lllllll}{5}&{5}&{5}&{5}&{5}&{6}&{4}\end{array}]{} & 1.5 & 27 & 0.48 & 0.6 & 0.69 & 1000 \\
\hline \multirow{4}{*}{11} & & \multirow{5}{*}{1.8} & \multirow{5}{*}{28} & 0.6 & 0.6 & 0.68 & 1000 \\
\hline & & & & 0.48 & 0.54 & 0.69 & 1000 \\
\hline & & & & 0.48 & 0.48 & 0.61 & 1000 \\
\hline & & & & 0.42 & 0.54 & 0.6633333 & 1000 \\
\hline & & & & 0.48 & 0.54 & 0.62 & 1000 \\
\hline \multirow{4}{*}{12} & & \multirow{4}{*}{2} & \multirow{4}{*}{29} & 0.54 & 0.54 & 0.63 & 1000 \\
\hline & & & & 0.42 & 0.54 & 0.6666667 & 1000 \\
\hline & & & & 0.54 & 0.6 & 0.5633333 & 1000 \\
\hline & & & & 0.36 & 0.54 & 0.5566667 & 1000 \\
\hline \multirow{2}{*}{13} & \multirow{2}{*}[\begin{array}{lllllll}{6}&{6}&{6}&{6}&{6}&{4}&{7}\end{array}]{} & \multirow{2}{*}{2} & \multirow{2}{*}{32} & 0.45 & 0.55 & 0.6 & 1000 \\
\hline & & & & 0.35 & 0.55 & 0.6714286 & 1000 \\
\hline \multirow{4}{*}{14} & \multirow{4}{*}[\begin{array}{lllllll}{6}&{6}&{5}&{5}&{6}&{4}&{7}\end{array}]{} & \multirow{4}{*}{2} & \multirow{4}{*}{32} & 0.44 & 0.52 & 0.6171429 & 1000 \\
\hline & & & & 0.42 & 0.57 & 0.6914286 & 1000 \\
\hline & & & & 0.39 & 0.59 & 0.6757143 & 1000 \\
\hline & & & & 0.48 & 0.58 & 0.63 & 1000 \\
\hline 15 & {$\left[\begin{array}{lllllll}5 & 5 & 6 & 6 & 6 & 4 & 7\end{array}\right]$} & 2 & 32 & 0.37 & 0.61 & 0.6857143 & 1000 \\
\hline
\end{tabular}

Pr. no.: problem number.

TABLE 12: Best bin combinations for dimension $X_{1}$.

\begin{tabular}{|c|c|c|c|c|c|c|c|c|c|c|c|c|c|c|c|c|c|c|c|c|c|c|c|c|c|c|c|c|c|c|c|c|c|c|c|c|c|c|c|c|c|}
\hline Pr. no. & & & & & & & & & & & & & & est & bin & com & bin & & & & & & & & & & & & & in & $\mathrm{oml}$ & ina & ion & & & & & & & & \\
\hline & 2 & 2 & 1 & 2 & 2 & 2 & 3 & 3 & 2 & 2 & 1 & 2 & 2 & 2 & 2 & & & & & & & & & & & & & & & & 1 & 1 & & & & & & & & & \\
\hline 1 & 1 & 2 & 3 & 2 & 3 & 2 & 2 & 2 & 2 & 2 & 2 & 2 & 2 & 2 & 2 & & & & & & & & & & & & & & & & 2 & 3 & & & & & & & & & \\
\hline & 2 & 1 & 2 & 2 & 3 & 2 & 2 & 1 & 2 & 3 & 3 & 3 & 3 & 3 & 3 & & 2 & & & & & & & & & & & & & & 1 & & 2 & 2 & 3 & 1 & & & & & \\
\hline 2 & 2 & 3 & 2 & 2 & 2 & 3 & 3 & 2 & 2 & 2 & 3 & 1 & 1 & 1 & 1 & & 2 & & & & 2 & & & & & & & & & & 1 & & 3 & 2 & 1 & 2 & & & & & \\
\hline & 3 & 1 & 1 & 2 & 3 & 3 & 2 & 2 & 2 & 2 & 2 & 2 & 2 & 2 & 2 & & 2 & & & & 2 & & & & & & & & & & 2 & & 1 & 2 & 2 & 2 & & & & & \\
\hline & 3 & 2 & 1 & 2 & 2 & 3 & 4 & 3 & 4 & 3 & 3 & 3 & 4 & 3 & 3 & & 3 & & & & 3 & & & & & & & & & & 3 & & 3 & 3 & 3 & 3 & 3 & 1 & 3 & & \\
\hline & 3 & 2 & 1 & 3 & 3 & 2 & 3 & 2 & 2 & 4 & 3 & 4 & 4 & 4 & 3 & 3 & 3 & & & & 3 & & & & & & & & & & & & 3 & 3 & 3 & 3 & 3 & 2 & 3 & & \\
\hline 3 & 1 & 3 & 2 & 2 & 3 & 2 & 3 & 4 & 3 & 4 & 4 & 2 & 2 & 2 & 1 & 2 & 2 & & & & 1 & & & & & & & & & & & & 2 & 2 & 1 & 1 & 3 & 3 & 3 & & \\
\hline & 2 & 3 & 3 & 3 & 3 & 1 & 3 & 2 & 4 & 4 & 3 & 2 & 2 & 2 & 2 & 2 & 2 & & & & 2 & & & & & & & & & & & 2 & 2 & 2 & 2 & 2 & 2 & 2 & 2 & & \\
\hline & 3 & 3 & 3 & 2 & 3 & 3 & 2 & 4 & 2 & 2 & 1 & 3 & 4 & 1 & 4 & 3 & 3 & & & & & & & & & & & & & & & 3 & 4 & 1 & 1 & 1 & 4 & 2 & 2 & 1 & 3 \\
\hline & 2 & 3 & 1 & 2 & 4 & 2 & 2 & 4 & 2 & 3 & 4 & 3 & 3 & 4 & 4 & 3 & 3 & & & 3 & 3 & & & & & & & & & & & & 4 & 3 & 3 & 3 & 3 & 3 & 2 & 3 & 3 \\
\hline 4 & 2 & 2 & 1 & 3 & 2 & 2 & 3 & 2 & 3 & 3 & 4 & 3 & 3 & 3 & 3 & 3 & 3 & & & & 3 & & & & & & & & & & & & 3 & 3 & 3 & 3 & 3 & 2 & 2 & 4 & 3 \\
\hline & 3 & 2 & 2 & 2 & 2 & 2 & 3 & 2 & 3 & 3 & 3 & 4 & 4 & 4 & 1 & 2 & 2 & & & & & & & & & & & & & & & & 2 & 2 & 2 & 2 & 2 & 2 & 2 & 3 & 4 \\
\hline & 3 & 2 & 2 & 3 & 3 & 2 & 4 & 4 & 3 & 3 & 5 & 3 & 5 & 4 & 2 & 4 & 1 & & & & & & & & & & & & & & & & 1 & 4 & 2 & 5 & 4 & 4 & 4 & 4 & \\
\hline 5 & 1 & 5 & 3 & 4 & 3 & 4 & 2 & 3 & 3 & 4 & 3 & 3 & 3 & 2 & 4 & 4 & 4 & & & & & & & & & & & & & & & & 4 & 4 & 2 & 2 & 4 & 2 & 4 & 4 & \\
\hline & 3 & 5 & 3 & 3 & 5 & 4 & 4 & 2 & 2 & 5 & 2 & 4 & 1 & 5 & 4 & 2 & & & & & & & & & & & & & & & & 1 & 4 & 1 & 4 & 4 & 4 & 2 & 2 & 2 & \\
\hline & 3 & 2 & 3 & 1 & 3 & 3 & 2 & 2 & 3 & 3 & 2 & 4 & 3 & 2 & 2 & 2 & 3 & & & & & & & & & & & & & & & & 4 & 3 & 2 & 4 & 4 & 4 & 3 & 2 & 1 \\
\hline 6 & 1 & 3 & 3 & 3 & 3 & 2 & 2 & 4 & 3 & 2 & 4 & 2 & 3 & 2 & 1 & 3 & 2 & & & & 2 & & & & & & & & & & & 2 & 2 & 2 & 2 & 2 & 2 & 1 & 1 & 1 & 3 \\
\hline & 2 & 2 & 3 & 3 & 4 & 3 & 2 & 3 & 3 & 4 & 2 & 1 & 2 & 2 & 3 & 2 & & & & & 3 & & & & & & & & & & & 3 & 3 & 3 & 3 & 3 & 3 & 3 & 3 & 3 & 2 \\
\hline & 1 & 4 & 3 & 3 & 4 & 4 & 2 & 4 & 2 & 3 & 4 & 3 & 3 & 3 & 4 & 5 & 2 & & & & 5 & & & & & & & & & & & & 3 & 5 & 2 & 5 & 2 & 4 & 4 & 2 & 2 \\
\hline & 3 & 3 & 4 & 2 & 2 & 5 & 3 & 3 & 3 & 4 & 4 & 3 & 4 & 3 & 4 & 4 & & & & & & & & & & & & & & & & 1 & 4 & 3 & 3 & 3 & 3 & 3 & 3 & 3 & 3 \\
\hline 7 & 3 & 1 & 3 & 4 & 2 & 4 & 2 & 3 & 3 & 3 & 4 & 4 & 5 & 4 & 4 & 4 & 2 & & & & 2 & & & & & & & & & & & & 4 & 2 & 2 & 2 & 2 & 2 & 2 & 2 & 2 \\
\hline & 3 & 3 & 3 & 1 & 3 & 3 & 4 & 2 & 4 & 2 & 5 & 2 & 3 & 3 & 3 & 2 & & & & & 2 & & & & & & & & & & & 3 & 3 & 2 & 2 & 2 & 2 & 2 & 2 & 2 & 4 \\
\hline & 3 & 2 & 3 & 3 & 3 & 2 & 4 & 4 & 5 & 2 & 5 & 3 & 3 & 4 & 4 & 4 & 4 & & & 4 & & & & & & & 5 & & & & & & 4 & 4 & 4 & 1 & 1 & 4 & 4 & 1 & 1 \\
\hline 8 & 3 & 4 & 5 & 3 & 3 & 2 & 3 & 3 & 3 & 3 & 3 & 1 & 3 & 3 & 2 & 2 & & & & & & & & & & & 4 & & & & & 2 & 2 & 2 & 2 & 2 & 2 & 2 & 2 & 2 & 1 \\
\hline & 2 & 3 & 4 & 5 & 4 & 3 & 4 & 4 & 4 & 1 & 3 & 4 & 3 & 3 & 3 & 3 & & & & & 1 & & & & & & 3 & & & & & 3 & 3 & 3 & 3 & 1 & 3 & 3 & 3 & 3 & 3 \\
\hline & 3 & 5 & 4 & 3 & 3 & 4 & 3 & 4 & 3 & 2 & 4 & 3 & 1 & 3 & 3 & 2 & & & & & & & & & & & 2 & & & & & 2 & 2 & 3 & 2 & 2 & 2 & 2 & 2 & 2 & 2 \\
\hline 9 & 3 & 4 & 2 & 3 & 3 & 4 & 4 & 1 & 4 & 3 & 2 & 3 & 5 & 3 & 3 & 2 & & & & & 4 & & & & & & 3 & & & & & 4 & 1 & 3 & 4 & 4 & 2 & 2 & 2 & 2 & 2 \\
\hline & 4 & 3 & 3 & 3 & 4 & 3 & 3 & 5 & 2 & 2 & 3 & 4 & 1 & 5 & 2 & 4 & & & & & & & & & & & 2 & & & 2 & & 1 & 4 & 4 & 4 & 4 & 2 & 2 & 2 & 2 & 2 \\
\hline 10 & 3 & 3 & 5 & 4 & 2 & 4 & 2 & 3 & 3 & 3 & 4 & 5 & 2 & 3 & 3 & 2 & & & & & & & & & & & 2 & & & & & 3 & 2 & 1 & 2 & 2 & 2 & 2 & 2 & 2 & 2 \\
\hline & 2 & 2 & 3 & 3 & 4 & 5 & 5 & 5 & 3 & 4 & 3 & 4 & 4 & 3 & 3 & 3 & & & & & & & & & & & 1 & 3 & & & & 3 & 3 & 3 & 3 & 3 & 3 & 3 & 1 & 3 & 3 \\
\hline & 2 & 4 & 3 & 4 & 1 & 5 & 3 & 4 & 3 & 3 & 2 & 3 & 3 & 2 & 4 & 2 & & & & & 3 & & & & & & 2 & 2 & & & & 4 & 4 & 3 & 3 & 3 & 3 & 4 & 3 & 2 & 2 \\
\hline 11 & 3 & 3 & 4 & 4 & 5 & 3 & 4 & 3 & 3 & 2 & 3 & 4 & 2 & 2 & 3 & 3 & & & & & & & & & & & 2 & 2 & & & & 2 & 5 & 1 & 3 & 2 & 3 & 3 & 3 & 2 & 3 \\
\hline & 2 & 2 & 4 & 4 & 1 & 3 & 3 & 4 & 3 & 2 & 3 & 3 & 4 & 3 & 2 & 3 & & & & & & & & & & & 3 & 3 & & & & 3 & 2 & 3 & 3 & 5 & 5 & 4 & 4 & 3 & 3 \\
\hline & 4 & 3 & 3 & 1 & 4 & 3 & 5 & 4 & 4 & 3 & 4 & 2 & 3 & 3 & 3 & 5 & & & & & & & & & & & 4 & 2 & 2 & & & 4 & 4 & 2 & 2 & 2 & 4 & 2 & 2 & 4 & 4 \\
\hline & 2 & 3 & 4 & 2 & 3 & 4 & 3 & 4 & 4 & 3 & 4 & 3 & 3 & 3 & 3 & 3 & & & & & 5 & & & & & & 2 & 1 & 1 & & & 2 & 2 & 5 & 2 & 5 & 3 & 5 & 3 & 3 & 1 \\
\hline 12 & 2 & 4 & 5 & 4 & 2 & 3 & 3 & 2 & 3 & 2 & 5 & 3 & 3 & 4 & 4 & 3 & 4 & & & & 4 & & & & & & 3 & 3 & 3 & & & 4 & 1 & 3 & 3 & 4 & 3 & 3 & 3 & 3 & 3 \\
\hline & 5 & 4 & 3 & 4 & 2 & 2 & 3 & 4 & 3 & 3 & 4 & 4 & 2 & 3 & 3 & 2 & & & & & & & & & & & 1 & 1 & 1 & & & 2 & 2 & 2 & 2 & 2 & 2 & 2 & 2 & 2 & 2 \\
\hline & 3 & 3 & 2 & 5 & 3 & 5 & 3 & 3 & 1 & 3 & 4 & 4 & 4 & 4 & 3 & 2 & 3 & 4 & & & & & & & & & 4 & 4 & 4 & & & & & 2 & 2 & 4 & 2 & 4 & 4 & 4 & 2 \\
\hline
\end{tabular}


TABle 12: Continued.

\begin{tabular}{|c|c|c|c|c|c|c|c|c|c|c|c|c|c|c|c|c|c|c|c|c|c|c|c|c|c|c|c|c|c|c|c|}
\hline Pr. no. & \multicolumn{31}{|c|}{ Best bin combinations } \\
\hline 13 & 5 & 4 & 4 & 2 & 4 & 4 & 4 & 3 & 4 & 2 & 3 & 6 & 5 & 6 & 3 & 5 & 3 & 3 & 3 & 3 & 3 & 2 & 3 & 3 & 3 & 3 & 3 & 3 & 3 & 2 & 1 \\
\hline 13 & 4 & 3 & 3 & 2 & 4 & 4 & 3 & 3 & 2 & 2 & 5 & 6 & 5 & 5 & 5 & 3 & 5 & 3 & 5 & 5 & 5 & 5 & 3 & 3 & 3 & 3 & 3 & 1 & 3 & 2 & 2 \\
\hline \multirow{4}{*}{14} & 3 & 4 & 3 & 4 & 1 & 4 & 3 & 2 & 5 & 4 & 3 & 5 & 5 & 6 & 3 & 2 & 4 & 3 & 4 & 6 & 3 & 3 & 3 & 3 & 3 & 3 & 2 & J & 2 & 2 & 2 \\
\hline & 3 & 4 & 4 & 3 & 3 & 4 & 5 & 3 & 5 & 5 & 6 & 2 & 4 & 4 & 3 & 4 & 3 & 2 & 3 & 6 & 2 & 1 & 6 & 3 & 5 & 3 & 3 & 3 & 3 & 2 & 2 \\
\hline & 2 & 4 & 4 & 2 & 4 & 5 & 5 & 3 & 3 & 3 & 4 & 3 & 4 & 4 & 4 & 3 & 6 & 5 & 4 & 3 & 3 & 3 & 3 & 4 & 6 & 6 & 5 & 4 & 1 & 4 & 4 \\
\hline & 5 & 5 & 4 & 4 & 4 & 4 & 5 & 6 & 4 & 6 & 2 & 4 & 3 & 3 & 3 & 3 & 3 & 3 & 2 & 3 & 3 & 3 & 3 & 3 & 2 & 2 & 2 & 2 & 2 & 2 & 1 \\
\hline 15 & 3 & 1 & 5 & 5 & 2 & 3 & 3 & 4 & 3 & 4 & 3 & 4 & 5 & 4 & 4 & 3 & 4 & 4 & 4 & 4 & 3 & 3 & 3 & 3 & 3 & 3 & 3 & 3 & 3 & 3 & 3 \\
\hline
\end{tabular}

TABle 13: Best bin combinations for dimension $X_{2 a}$.

\begin{tabular}{|c|c|c|c|c|c|c|c|c|c|c|c|c|c|c|c|c|c|c|c|c|c|c|c|c|c|c|c|c|c|c|c|c|}
\hline Pr. no. & \multicolumn{32}{|c|}{ Best bin combinations } \\
\hline \multirow{2}{*}{1} & 2 & 2 & 1 & 3 & 2 & 1 & 2 & 2 & 2 & 2 & 2 & 1 & 2 & 1 & 2 & 2 & 2 & & & & & & & & & & & & & & & \\
\hline & 3 & 2 & 1 & 1 & 3 & 2 & 2 & 2 & 2 & 2 & 2 & 3 & 2 & 2 & 2 & 2 & 1 & & & & & & & & & & & & & & & \\
\hline \multirow{3}{*}{2} & 3 & 2 & 2 & 1 & 1 & 3 & 2 & 2 & 2 & 2 & 2 & 3 & 3 & 2 & 2 & 2 & 1 & 2 & 2 & 2 & 2 & & & & & & & & & & & \\
\hline & 2 & 1 & 3 & 2 & 2 & 2 & 2 & 2 & 2 & 2 & 1 & 2 & 1 & 2 & 2 & 1 & 3 & 2 & 1 & 2 & 2 & & & & & & & & & & & \\
\hline & 3 & 1 & 2 & 1 & 1 & 2 & 2 & 1 & 3 & 2 & 2 & 2 & 2 & 2 & 1 & 1 & 2 & 2 & 2 & 2 & 3 & & & & & & & & & & & \\
\hline \multirow{4}{*}{3} & 3 & 3 & 4 & 2 & 2 & 1 & 2 & 2 & 1 & 3 & 3 & 1 & 2 & 2 & 3 & 2 & 2 & 3 & 4 & 4 & 2 & 4 & 3 & 3 & & & & & & & & \\
\hline & 2 & 2 & 1 & 4 & 4 & 3 & 3 & 3 & 1 & 3 & 3 & 3 & 3 & 3 & 3 & 3 & 3 & 3 & 3 & 1 & 1 & 1 & 1 & 2 & & & & & & & & \\
\hline & 3 & 2 & 2 & 2 & 3 & 2 & 2 & 3 & 1 & 3 & 3 & 1 & 1 & 3 & 2 & 4 & 2 & 4 & 4 & 4 & 4 & 3 & 3 & 2 & & & & & & & & \\
\hline & 3 & 2 & 1 & 3 & 2 & 3 & 2 & 3 & 4 & 3 & 1 & 2 & 2 & 1 & 2 & 3 & 4 & 3 & 3 & 3 & 3 & 3 & 2 & 3 & & & & & & & & \\
\hline \multirow{4}{*}{4} & 3 & 3 & 4 & 1 & 2 & 2 & 2 & 4 & 3 & 3 & 3 & 3 & 3 & 3 & 3 & 3 & 3 & 3 & 3 & 3 & 3 & 3 & 1 & 2 & 2 & 2 & & & & & & \\
\hline & 2 & 3 & 2 & 2 & 3 & 4 & 2 & 2 & 3 & 1 & 2 & 3 & 3 & 3 & 3 & 3 & 3 & 1 & 2 & 1 & 1 & 1 & 3 & 2 & 3 & 1 & & & & & & \\
\hline & 1 & 4 & 2 & 3 & 3 & 2 & 2 & 3 & 3 & 2 & 2 & 2 & 2 & 2 & 2 & 1 & 2 & 2 & 1 & 2 & 1 & 1 & 2 & 2 & 2 & 3 & & & & & & \\
\hline & 1 & 3 & 2 & 3 & 2 & 2 & 3 & 4 & 3 & 2 & 2 & 2 & 2 & 2 & 2 & 2 & 2 & 2 & 2 & 2 & 2 & 2 & 3 & 3 & 1 & 2 & & & & & & \\
\hline \multirow{3}{*}{5} & 3 & 1 & 2 & 3 & 3 & 4 & 4 & 2 & 2 & 2 & 2 & 2 & 2 & 1 & 2 & 2 & 2 & 2 & 2 & 2 & 2 & 2 & 2 & 2 & 3 & & & & & & & \\
\hline & 1 & 3 & 2 & 2 & 2 & 3 & 4 & 2 & 1 & 3 & 2 & 3 & 3 & 1 & 2 & 3 & 3 & 1 & 2 & 3 & 4 & 2 & 3 & 3 & 3 & & & & & & & \\
\hline & 1 & 2 & 2 & 3 & 1 & 2 & 3 & 3 & 3 & 2 & 4 & 4 & 4 & 4 & 4 & 2 & 2 & 2 & 2 & 2 & 2 & 2 & 2 & 2 & 2 & & & & & & & \\
\hline \multirow{3}{*}{6} & 3 & 2 & 3 & 3 & 3 & 3 & 2 & 1 & 4 & 4 & 4 & 2 & 4 & 4 & 2 & 2 & 2 & 2 & 2 & 2 & 2 & 2 & 2 & 2 & 2 & 2 & & & & & & \\
\hline & 4 & 3 & 3 & 3 & 2 & 3 & 3 & 2 & 2 & 3 & 2 & 2 & 3 & 2 & 4 & 2 & 2 & 4 & 1 & 2 & 2 & 1 & 1 & 1 & 1 & 3 & & & & & & \\
\hline & 2 & 2 & 2 & 4 & 4 & 1 & 3 & 3 & 3 & 3 & 3 & 3 & 3 & 3 & 3 & 3 & 3 & 3 & 3 & 1 & 1 & 1 & 1 & 1 & 1 & 3 & & & & & & \\
\hline \multirow{4}{*}{7} & 3 & 2 & 3 & 2 & 3 & 2 & 2 & 3 & 4 & 3 & 5 & 3 & 1 & 4 & 3 & 4 & 4 & 3 & 3 & 3 & 3 & 3 & 1 & 3 & 3 & 1 & & & & & & \\
\hline & 2 & 3 & 2 & 3 & 3 & 2 & 2 & 4 & 5 & 5 & 1 & 3 & 2 & 5 & 4 & 4 & 4 & 4 & 2 & 4 & 2 & 1 & 1 & 1 & 1 & 1 & & & & & & \\
\hline & 3 & 2 & 5 & 2 & 5 & 1 & 5 & 4 & 4 & 4 & 2 & 4 & 4 & 4 & 4 & 2 & 2 & 4 & 4 & 4 & 4 & 2 & 2 & 1 & 1 & 1 & & & & & & \\
\hline & 2 & 4 & 5 & 3 & 3 & 3 & 2 & 3 & 3 & 2 & 3 & 3 & 3 & 3 & 3 & 3 & 1 & 3 & 3 & 3 & 3 & 1 & 1 & 1 & 1 & 1 & & & & & & \\
\hline & 4 & 2 & 3 & 2 & 3 & 2 & 1 & 3 & 1 & 3 & 3 & 1 & 2 & 2 & 3 & 2 & 3 & 3 & 2 & 3 & 2 & 2 & 2 & 1 & 1 & 1 & 2 & & & & & \\
\hline 8 & 2 & 3 & 2 & 4 & 2 & 3 & 1 & 3 & 3 & 3 & 3 & 2 & 2 & 2 & 2 & 1 & 3 & 2 & 2 & 1 & 2 & 2 & 1 & 2 & 2 & 2 & 2 & & & & & \\
\hline & 3 & 3 & 2 & 2 & 3 & 4 & 1 & 4 & 2 & 2 & 2 & 2 & 2 & 2 & 2 & 1 & 2 & 2 & 2 & 2 & 2 & 1 & 1 & 2 & 2 & 2 & 3 & & & & & \\
\hline & 3 & 4 & 2 & 3 & 1 & 2 & 2 & 3 & 3 & 2 & 3 & 1 & 2 & 2 & 3 & 3 & 2 & 2 & 4 & 4 & 3 & 2 & 2 & 2 & 2 & 2 & 2 & & & & & \\
\hline 9 & 4 & 2 & 2 & 2 & 2 & 1 & 3 & 3 & 3 & 3 & 3 & 2 & 3 & 3 & 2 & 3 & 3 & 2 & 2 & 2 & 2 & 2 & 2 & 2 & 2 & 2 & 2 & & & & & \\
\hline & 3 & 2 & 3 & 3 & 3 & 2 & 4 & 2 & 1 & 2 & 3 & 4 & 3 & 3 & 4 & 3 & 3 & 3 & 1 & 1 & 1 & 3 & 3 & 3 & 3 & 1 & 1 & & & & & \\
\hline 10 & 1 & 3 & 2 & 5 & 3 & 4 & 3 & 4 & 1 & 2 & 3 & 3 & 3 & 3 & 3 & 2 & 3 & 3 & 2 & 2 & 2 & 3 & 3 & 2 & 2 & 2 & 2 & & & & & \\
\hline & 3 & 3 & 3 & 2 & 2 & 3 & 5 & 4 & 3 & 1 & 2 & 5 & 4 & 4 & 4 & 4 & 2 & 2 & 4 & 4 & 2 & 4 & 4 & 4 & 1 & 1 & 1 & 1 & & & & \\
\hline & 4 & 4 & 5 & 2 & 3 & 3 & 2 & 3 & 3 & 3 & 2 & 3 & 3 & 2 & 3 & 1 & 2 & 1 & 2 & 3 & 2 & 2 & 2 & 2 & 2 & 1 & 2 & 2 & & & & \\
\hline 1 & 4 & 1 & 2 & 2 & 3 & 2 & 3 & 5 & 3 & 3 & 4 & 3 & 4 & 3 & 3 & 1 & 4 & 4 & 3 & 3 & 4 & 4 & 4 & 3 & 3 & 3 & 3 & 3 & & & & \\
\hline & 3 & 3 & 3 & 4 & 5 & 1 & 4 & 2 & 2 & 3 & 5 & 3 & 3 & 3 & 4 & 5 & 5 & 4 & 4 & 3 & 4 & 3 & 3 & 4 & 4 & 4 & 4 & 4 & & & & \\
\hline & 2 & 3 & 2 & 3 & 2 & 3 & 4 & 1 & 5 & 2 & 3 & 2 & 1 & 4 & 3 & 3 & 4 & 3 & 4 & 4 & 4 & 3 & 3 & 3 & 3 & 3 & 3 & 3 & 3 & & & \\
\hline & 1 & 5 & 4 & 2 & 4 & 3 & 3 & 4 & 3 & 3 & 3 & 3 & 2 & 2 & 3 & 3 & 2 & 2 & 3 & 3 & 3 & 3 & 3 & 3 & 2 & 2 & 2 & 2 & 2 & & & \\
\hline 12 & 3 & 2 & 5 & 3 & 3 & 3 & 4 & 2 & 1 & 3 & 4 & 4 & 3 & 5 & 4 & 5 & 4 & 4 & 3 & 4 & 3 & 4 & 1 & 3 & 3 & 3 & 3 & 3 & 1 & & & \\
\hline & 4 & 2 & 3 & 3 & 5 & 3 & 4 & 3 & 4 & 4 & 2 & 2 & 2 & 3 & 3 & 2 & 4 & 3 & 4 & 3 & 4 & 4 & 1 & 2 & 2 & 1 & 1 & 1 & 1 & & & \\
\hline & 3 & 3 & 2 & 4 & 3 & 3 & 4 & 2 & 3 & 2 & 5 & 2 & 4 & 3 & 1 & 4 & 3 & 3 & 3 & 5 & 4 & 4 & 2 & 5 & 2 & 5 & 2 & 4 & 5 & & & \\
\hline & 3 & 4 & 3 & 4 & 5 & 4 & 4 & 3 & 3 & 3 & 4 & 1 & 3 & 2 & 2 & 2 & 3 & 6 & 1 & 6 & 4 & 2 & 3 & 3 & 2 & 2 & 2 & 2 & 2 & 2 & 2 & \\
\hline 1 & 3 & 3 & 6 & 4 & 4 & 2 & 3 & 5 & 4 & 5 & 4 & 1 & 3 & 6 & 5 & 5 & 4 & 1 & 5 & 5 & 4 & 2 & 2 & 2 & 2 & 2 & 2 & 2 & 2 & 2 & 2 & 2 \\
\hline & 4 & 4 & 4 & 3 & 3 & 5 & 2 & 4 & 3 & 2 & 5 & 1 & 2 & 3 & 6 & 3 & 3 & 1 & 4 & 4 & 3 & 5 & 4 & 6 & 5 & 2 & 5 & 5 & 3 & 2 & 3 & \\
\hline & 3 & 4 & 1 & 5 & 4 & 4 & 4 & 3 & 3 & 5 & 3 & 5 & 1 & 2 & 3 & 3 & 6 & 5 & 5 & 5 & 5 & 5 & 4 & 4 & 4 & 4 & 3 & 4 & 3 & 3 & 3 & \\
\hline 14 & 5 & 4 & 4 & 3 & 5 & 2 & 6 & 2 & 4 & 4 & 3 & 4 & 4 & 3 & 3 & 3 & 3 & 3 & 3 & 3 & 3 & 3 & 3 & 1 & 1 & 3 & 3 & 3 & 3 & 3 & 3 & \\
\hline & 2 & 4 & 4 & 3 & 4 & 4 & 3 & 6 & 3 & 1 & 2 & 6 & 1 & 6 & 2 & 5 & 5 & 3 & 5 & 5 & 5 & 3 & 3 & 3 & 3 & 3 & 3 & 1 & 3 & 3 & 3 & 3 \\
\hline 15 & 1 & 2 & 3 & 2 & 4 & 3 & 2 & 2 & 1 & 5 & 4 & 3 & 3 & 3 & 3 & 2 & 1 & 3 & 3 & 3 & 3 & 3 & 3 & 3 & 2 & 3 & 3 & 3 & 3 & 1 & 1 & \\
\hline
\end{tabular}


TABle 14: Best bin combinations for dimension $X_{2 b}$.

\begin{tabular}{|c|c|c|c|c|c|c|c|c|c|c|c|c|c|c|c|c|c|c|c|c|c|c|c|c|c|c|c|c|c|c|c|c|}
\hline Pr. no. & \multicolumn{32}{|c|}{ Best bin combinations } \\
\hline \multirow{2}{*}{1} & 3 & 3 & 2 & 2 & 2 & 1 & 2 & 2 & 2 & 2 & 2 & 2 & 2 & 2 & 2 & 2 & 3 & & & & & & & & & & & & & & & \\
\hline & 2 & 2 & 2 & 2 & 2 & 2 & 3 & 1 & 2 & 2 & 2 & 3 & 3 & 1 & 1 & 1 & 3 & & & & & & & & & & & & & & & \\
\hline \multirow{3}{*}{2} & 2 & 3 & 2 & 3 & 2 & 1 & 2 & 3 & 2 & 2 & 2 & 2 & 2 & 2 & 2 & 2 & 3 & 2 & 2 & 2 & 2 & & & & & & & & & & & \\
\hline & 2 & 2 & 3 & 3 & 2 & 1 & 2 & 2 & 2 & 2 & 2 & 1 & 2 & 2 & 2 & 2 & 2 & 3 & 2 & 2 & 2 & & & & & & & & & & & \\
\hline & 1 & 2 & 3 & 2 & 2 & 3 & 3 & 2 & 2 & 2 & 2 & 2 & 2 & 2 & 2 & 2 & 2 & 3 & 2 & 2 & 2 & & & & & & & & & & & \\
\hline \multirow{4}{*}{3} & 2 & 3 & 3 & 3 & 2 & 1 & 3 & 3 & 4 & 2 & 3 & 3 & 3 & 4 & 2 & 3 & 3 & 3 & 3 & 3 & 3 & 3 & 2 & 4 & & & & & & & & \\
\hline & 3 & 3 & 3 & 3 & 3 & 2 & 2 & 4 & 4 & 3 & 1 & 1 & 3 & 2 & 4 & 2 & 2 & 2 & 2 & 2 & 2 & 2 & 3 & 2 & & & & & & & & \\
\hline & 4 & 3 & 3 & 3 & 2 & 3 & 3 & 4 & 3 & 1 & 4 & 3 & 4 & 3 & 3 & 3 & 3 & 3 & 3 & 3 & 1 & 1 & 3 & 3 & & & & & & & & \\
\hline & 3 & 3 & 4 & 3 & 3 & 2 & 2 & 3 & 3 & 2 & 3 & 1 & 3 & 2 & 4 & 3 & 2 & 4 & 4 & 2 & 2 & 2 & 3 & 2 & & & & & & & & \\
\hline \multirow{4}{*}{4} & 3 & 3 & 3 & 2 & 3 & 3 & 3 & 4 & 2 & 3 & 3 & 4 & 3 & 4 & 4 & 3 & 4 & 4 & 4 & 4 & 1 & 1 & 1 & 4 & 4 & 3 & & & & & & \\
\hline & 3 & 3 & 2 & 2 & 4 & 4 & 3 & 1 & 3 & 3 & 4 & 3 & 3 & 3 & 3 & 3 & 3 & 3 & 3 & 3 & 3 & 3 & 3 & 3 & 3 & 4 & & & & & & \\
\hline & 3 & 4 & 3 & 3 & 2 & 3 & 4 & 3 & 3 & 2 & 3 & 4 & 3 & 3 & 2 & 1 & 3 & 3 & 3 & 3 & 3 & 1 & 1 & 3 & 2 & 3 & & & & & & \\
\hline & 4 & 3 & 2 & 4 & 3 & 3 & 3 & 3 & 2 & 2 & 3 & 3 & 1 & 3 & 3 & 2 & 3 & 3 & 3 & 3 & 2 & 2 & 3 & 3 & 2 & 2 & & & & & & \\
\hline \multirow{3}{*}{5} & 4 & 4 & 2 & 4 & 3 & 5 & 3 & 3 & 4 & 4 & 3 & 4 & 4 & 3 & 3 & 4 & 1 & 4 & 4 & 4 & 4 & 4 & 4 & 4 & 2 & & & & & & & \\
\hline & 3 & 5 & 4 & 4 & 3 & 3 & 4 & 4 & 3 & 1 & 3 & 5 & 4 & 3 & 3 & 3 & 4 & 4 & 3 & 2 & 4 & 4 & 4 & 4 & 3 & & & & & & & \\
\hline & 2 & 3 & 1 & 3 & 4 & 5 & 4 & 4 & 3 & 3 & 4 & 3 & 4 & 3 & 4 & 4 & 3 & 2 & 4 & 4 & 4 & 4 & 4 & 4 & 4 & & & & & & & \\
\hline \multirow{3}{*}{6} & 2 & 4 & 2 & 3 & 3 & 3 & 2 & 3 & 3 & 3 & 3 & 3 & 1 & 3 & 3 & 3 & 3 & 3 & 3 & 3 & 3 & 3 & 3 & 3 & 3 & 4 & & & & & & \\
\hline & 1 & 2 & 2 & 3 & 4 & 4 & 3 & 3 & 4 & 3 & 3 & 4 & 3 & 3 & 3 & 3 & 3 & 3 & 3 & 3 & 3 & 3 & 3 & 3 & 3 & 3 & & & & & & \\
\hline & 2 & 1 & 3 & 3 & 3 & 3 & 4 & 4 & 4 & 3 & 4 & 3 & 3 & 3 & 3 & 3 & 3 & 3 & 3 & 3 & 3 & 3 & 3 & 3 & 3 & 3 & & & & & & \\
\hline \multirow{4}{*}{7} & 3 & 4 & 3 & 3 & 3 & 3 & 3 & 3 & 1 & 4 & 2 & 3 & 3 & 3 & 2 & 3 & 2 & 3 & 3 & 2 & 2 & 2 & 2 & 2 & 2 & 2 & & & & & & \\
\hline & 3 & 3 & 2 & 3 & 3 & 3 & 4 & 3 & 3 & 4 & 3 & 3 & 3 & 3 & 2 & 3 & 3 & 2 & 1 & 4 & 3 & 4 & 2 & 3 & 2 & 3 & & & & & & \\
\hline & 3 & 2 & 1 & 2 & 2 & 3 & 4 & 3 & 3 & 3 & 3 & 3 & 2 & 3 & 3 & 4 & 4 & 2 & 4 & 2 & 2 & 2 & 2 & 2 & 2 & 2 & & & & & & \\
\hline & 2 & 3 & 3 & 4 & 3 & 2 & 2 & 3 & 3 & 3 & 3 & 3 & 3 & 3 & 4 & 1 & 2 & 4 & 4 & 1 & 4 & 4 & 4 & 4 & 4 & 3 & & & & & & \\
\hline & 2 & 4 & 2 & 3 & 3 & 3 & 4 & 4 & 1 & 3 & 3 & 4 & 3 & 4 & 3 & 4 & 5 & 4 & 5 & 4 & 4 & 4 & 4 & 4 & 4 & 4 & 3 & & & & & \\
\hline 8 & 2 & 4 & 4 & 3 & 4 & 3 & 5 & 5 & 3 & 5 & 3 & 3 & 3 & 3 & 3 & 3 & 3 & 3 & 1 & 3 & 3 & 3 & 3 & 3 & 3 & 3 & 4 & & & & & \\
\hline & 3 & 4 & 2 & 4 & 4 & 2 & 3 & 3 & 5 & 5 & 3 & 3 & 4 & 4 & 3 & 1 & 4 & 3 & 4 & 4 & 3 & 3 & 3 & 3 & 3 & 3 & 3 & & & & & \\
\hline & 3 & 3 & 4 & 4 & 4 & 3 & 2 & 3 & 3 & 5 & 4 & 4 & 4 & 4 & 5 & 2 & 3 & 3 & 2 & 3 & 3 & 3 & 2 & 2 & 1 & 2 & 2 & & & & & \\
\hline 9 & 4 & 2 & 3 & 3 & 4 & 4 & 3 & 1 & 3 & 5 & 4 & 2 & 3 & 4 & 3 & 3 & 3 & 3 & 3 & 3 & 3 & 3 & 3 & 3 & 2 & 2 & 2 & & & & & \\
\hline & 2 & 3 & 4 & 3 & 4 & 4 & 3 & 4 & 4 & 3 & 3 & 4 & 3 & 3 & 3 & 5 & 5 & 5 & 3 & 3 & 3 & 1 & 3 & 3 & 3 & 3 & 3 & & & & & \\
\hline 10 & 4 & 3 & 4 & 3 & 4 & 4 & 4 & 3 & 5 & 4 & 3 & 3 & 3 & 3 & 2 & 5 & 4 & 2 & 4 & 4 & 1 & 4 & 2 & 4 & 4 & 2 & 2 & & & & & \\
\hline & 3 & 4 & 2 & 4 & 4 & 5 & 3 & 3 & 4 & 5 & 4 & 3 & 3 & 3 & 3 & 3 & 3 & 3 & 3 & 3 & 3 & 3 & 3 & 3 & 3 & 1 & 1 & 2 & & & & \\
\hline & 2 & 4 & 3 & 5 & 4 & 4 & 3 & 3 & 3 & 3 & 3 & 4 & 4 & 1 & 3 & 4 & 3 & 3 & 3 & 3 & 4 & 4 & 3 & 3 & 3 & 3 & 3 & 3 & & & & \\
\hline 1 & 2 & 4 & 3 & 5 & 3 & 3 & 4 & 3 & 4 & 5 & 3 & 4 & 4 & 1 & 4 & 4 & 3 & 4 & 4 & 4 & 4 & 3 & 3 & 3 & 4 & 3 & 3 & 3 & & & & \\
\hline & 3 & 5 & 3 & 3 & 4 & 4 & 4 & 2 & 3 & 3 & 4 & 3 & 4 & 4 & 3 & 4 & 3 & 4 & 1 & 3 & 4 & 4 & 4 & 4 & 4 & 4 & 4 & 4 & & & & \\
\hline & 5 & 3 & 4 & 3 & 4 & 4 & 4 & 3 & 2 & 4 & 4 & 4 & 3 & 4 & 3 & 4 & 4 & 3 & 3 & 1 & 3 & 3 & 3 & 3 & 2 & 3 & 2 & 2 & 2 & & & \\
\hline & 3 & 4 & 4 & 1 & 2 & 3 & 4 & 2 & 3 & 3 & 3 & 4 & 4 & 3 & 3 & 4 & 5 & 3 & 5 & 3 & 5 & 3 & 3 & 3 & 2 & 3 & 3 & 3 & 2 & & & \\
\hline 12 & 3 & 4 & 4 & 1 & 2 & 3 & 5 & 4 & 3 & 3 & 4 & 4 & 4 & 4 & 3 & 3 & 3 & 4 & 4 & 3 & 3 & 3 & 3 & 3 & 3 & 3 & 3 & 3 & 3 & & & \\
\hline & 2 & 4 & 4 & 3 & 2 & 3 & 3 & 3 & 3 & 4 & 3 & 5 & 3 & 3 & 3 & 5 & 3 & 5 & 5 & 3 & 2 & 3 & 3 & 3 & 3 & 3 & 3 & 3 & 1 & & & \\
\hline & 3 & 5 & 2 & 3 & 3 & 3 & 3 & 3 & 4 & 4 & 4 & 4 & 4 & 3 & 4 & 4 & 3 & 4 & 4 & 3 & 4 & 4 & 4 & 1 & 4 & 4 & 4 & 4 & 4 & & & \\
\hline 13 & 4 & 5 & 2 & 4 & 3 & 4 & 3 & 6 & 5 & 5 & 5 & 5 & 5 & 4 & 1 & 4 & 4 & 4 & 4 & 4 & 4 & 4 & 4 & 4 & 4 & 4 & 4 & 4 & 4 & 4 & 4 & $\overline{4}$ \\
\hline 13 & 3 & 3 & 6 & 4 & 5 & 5 & 4 & 5 & 4 & 5 & 4 & 4 & 4 & 4 & 4 & 2 & 3 & 4 & 3 & 4 & 4 & 1 & 4 & 4 & 4 & 4 & 4 & 4 & 4 & 4 & 4 & 4 \\
\hline & 3 & 2 & 1 & 3 & 4 & 3 & 5 & 4 & 4 & 3 & 4 & 3 & 4 & 3 & 4 & 4 & 3 & 3 & 4 & 4 & 3 & 4 & 3 & 4 & 4 & 4 & 3 & 4 & 4 & 4 & 4 & 4 \\
\hline 1 & 3 & 2 & 3 & 3 & 5 & 3 & 4 & 4 & 3 & 3 & 1 & 4 & 3 & 3 & 4 & 4 & 4 & 3 & 3 & 4 & 3 & 4 & 4 & 4 & 3 & 3 & 3 & 3 & 3 & 3 & 3 & 3 \\
\hline 12 & 5 & 1 & 2 & 3 & 4 & 3 & 4 & 3 & 3 & 3 & 4 & 4 & 3 & 3 & 3 & 4 & 4 & 4 & 4 & 3 & 4 & 4 & 4 & 4 & 4 & 4 & 4 & 4 & 4 & 4 & 4 & 4 \\
\hline & 4 & 1 & 5 & 2 & 3 & 4 & 3 & 2 & 4 & 4 & 4 & 4 & 3 & 3 & 4 & 3 & 3 & 4 & 4 & 4 & 3 & 4 & 4 & 4 & 4 & 4 & 2 & 2 & 2 & 2 & 2 & 2 \\
\hline 15 & 4 & 5 & 2 & 1 & 4 & 3 & 4 & 3 & 2 & 3 & 4 & 3 & 4 & 4 & 5 & 3 & 6 & 5 & 5 & 5 & 4 & 6 & 4 & 4 & 6 & 6 & 5 & 5 & 5 & 5 & 5 & \\
\hline
\end{tabular}

$\left(x_{i, j}\right)$ are considered to identify the new solution space $\left(N_{i, j}\right)$ :

$$
N_{i, j}=x_{i, j}+\varphi_{i, j}\left(x_{i, j}-x_{k, j}\right) .
$$

The replacement of employed bees is based on the evaluation results of randomly generated bees. The greedy selection process stated in Karaboga and Basturk [30] is used for this purpose. (d) Identification of new solutions instead of unemployed bees

The new bees are randomly generated based on roulette wheel selection stated in Nagarajan et al. [32] and are equal in numbers concerning unemployed bees, followed by the updation of unemployed bees with the newly generated bees using the greedy selection process. 
TABle 15: Best bin combinations for dimension $X_{3 a}$.

\begin{tabular}{|c|c|c|c|c|c|c|c|c|c|c|c|c|c|c|c|c|c|c|c|c|c|c|c|c|c|c|c|c|c|c|c|c|c|}
\hline Pr. no. & \multicolumn{33}{|c|}{ Best bin combinations } \\
\hline \multirow{2}{*}{1} & 2 & 2 & 2 & 2 & 2 & 2 & 1 & 2 & 2 & 3 & 3 & 3 & 3 & 3 & 3 & 3 & 1 & & & & & & & & & & & & & & & & \\
\hline & 2 & 1 & 3 & 2 & 2 & 2 & 2 & 2 & 2 & 2 & 2 & 2 & 2 & 2 & 2 & 2 & 2 & & & & & & & & & & & & & & & & \\
\hline \multirow{3}{*}{2} & 2 & 2 & 2 & 3 & 2 & 1 & 2 & 1 & 2 & 2 & 2 & 1 & 2 & 2 & 2 & 2 & 3 & 2 & 2 & 3 & 2 & & & & & & & & & & & & \\
\hline & 2 & 2 & 2 & 3 & 1 & 3 & 2 & 3 & 2 & 2 & 2 & 2 & 2 & 2 & 2 & 2 & 1 & 2 & 3 & 2 & 3 & & & & & & & & & & & & \\
\hline & 2 & 2 & 2 & 2 & 3 & 2 & 1 & 2 & 2 & 1 & 3 & 2 & 2 & 2 & 2 & 2 & 2 & 3 & 2 & 2 & 2 & & & & & & & & & & & & \\
\hline \multirow{4}{*}{3} & 3 & 3 & 2 & 2 & 3 & 3 & 1 & 3 & 4 & 2 & 2 & 2 & 2 & 2 & 2 & 2 & 2 & 2 & 2 & 2 & 1 & 1 & 1 & 2 & & & & & & & & & \\
\hline & 4 & 3 & 1 & 2 & 3 & 3 & 2 & 3 & 2 & 3 & 3 & 2 & 2 & 2 & 2 & 2 & 2 & 2 & 2 & 2 & 2 & 1 & 2 & 1 & & & & & & & & & \\
\hline & 3 & 3 & 3 & 4 & 3 & 3 & 2 & 2 & 2 & 2 & 2 & 2 & 2 & 2 & 2 & 1 & 2 & 2 & 1 & 1 & 1 & 2 & 2 & 3 & & & & & & & & & \\
\hline & 2 & 3 & 2 & 2 & 2 & 3 & 2 & 3 & 3 & 4 & 1 & 3 & 4 & 3 & 3 & 3 & 3 & 1 & 3 & 3 & 3 & 3 & 3 & 2 & & & & & & & & & \\
\hline \multirow{4}{*}{4} & 3 & 3 & 1 & 3 & 3 & 3 & 2 & 3 & 2 & 4 & 2 & 4 & 2 & 4 & 2 & 2 & 2 & 2 & 2 & 2 & 2 & 2 & 3 & 1 & 2 & 2 & & & & & & & \\
\hline & 3 & 3 & 3 & 2 & 4 & 2 & 1 & 2 & 3 & 2 & 2 & 2 & 3 & 4 & 3 & 3 & 3 & 3 & 3 & 3 & 3 & 3 & 3 & 2 & 4 & 2 & & & & & & & \\
\hline & 3 & 3 & 2 & 3 & 2 & 2 & 3 & 2 & 3 & 4 & 2 & 2 & 2 & 3 & 3 & 1 & 3 & 1 & 3 & 3 & 3 & 3 & 3 & 2 & 2 & 3 & & & & & & & \\
\hline & 2 & 3 & 1 & 2 & 2 & 3 & 3 & 3 & 2 & 3 & 2 & 3 & 3 & 2 & 2 & 2 & 4 & 3 & 3 & 4 & 4 & 4 & 4 & 2 & 2 & 1 & & & & & & & \\
\hline \multirow{3}{*}{5} & 3 & 2 & 2 & 3 & 3 & 2 & 3 & 3 & 4 & 2 & 1 & 4 & 4 & 2 & 2 & 4 & 2 & 2 & 2 & 1 & 2 & 2 & 2 & 1 & 1 & & & & & & & & \\
\hline & 2 & 2 & 2 & 4 & 3 & 3 & 2 & 1 & 3 & 2 & 3 & 2 & 3 & 2 & 3 & 3 & 2 & 2 & 2 & 2 & 2 & 2 & 2 & 2 & 2 & & & & & & & & \\
\hline & 2 & 1 & 3 & 3 & 1 & 4 & 3 & 4 & 2 & 1 & 2 & 3 & 2 & 2 & 3 & 2 & 3 & 2 & 3 & 3 & 2 & 2 & 2 & 2 & 1 & & & & & & & & \\
\hline \multirow{3}{*}{6} & 3 & 2 & 2 & 3 & 2 & 3 & 2 & 3 & 1 & 2 & 3 & 3 & 2 & 2 & 2 & 3 & 4 & 1 & 4 & 4 & 4 & 4 & 1 & 2 & 4 & 2 & & & & & & & \\
\hline & 3 & 2 & 2 & 4 & 1 & 3 & 3 & 3 & 2 & 2 & 2 & 3 & 3 & 2 & 3 & 3 & 3 & 3 & 3 & 3 & 3 & 3 & 3 & 3 & 2 & 3 & & & & & & & \\
\hline & 2 & 3 & 2 & 2 & 4 & 4 & 3 & 3 & 3 & 3 & 3 & 3 & 1 & 3 & 1 & 3 & 3 & 3 & 3 & 3 & 3 & 3 & 3 & 3 & 3 & 2 & & & & & & & \\
\hline \multirow{4}{*}{7} & 3 & 4 & 3 & 2 & 2 & 2 & 2 & 3 & 3 & 3 & 2 & 2 & 1 & 3 & 3 & 2 & 2 & 2 & 3 & 2 & 3 & 3 & 3 & 1 & 1 & 1 & & & & & & & \\
\hline & 2 & 2 & 2 & 2 & 3 & 2 & 4 & 2 & 3 & 3 & 3 & 3 & 3 & 3 & 3 & 3 & 3 & 3 & 3 & 3 & 1 & 3 & 3 & 3 & 1 & 1 & & & & & & & \\
\hline & 3 & 4 & 1 & 2 & 1 & 2 & 2 & 3 & 3 & 2 & 2 & 2 & 2 & 2 & 2 & 4 & 2 & 3 & 2 & 3 & 3 & 3 & 2 & 2 & 2 & 2 & & & & & & & \\
\hline & 1 & 2 & 2 & 3 & 4 & 2 & 3 & 3 & 3 & 2 & 3 & 2 & 1 & 2 & 2 & 2 & 2 & 3 & 2 & 3 & 3 & 3 & 4 & 4 & 1 & 2 & & & & & & & \\
\hline & 2 & 2 & 3 & 1 & 3 & 2 & 3 & 3 & 2 & 2 & 2 & 2 & 2 & 3 & 3 & 3 & 3 & 4 & 3 & 4 & 4 & 2 & 3 & 3 & 2 & 3 & 3 & & & & & & \\
\hline 8 & 2 & 3 & 2 & 2 & 3 & 4 & 3 & 2 & 2 & 3 & 3 & 1 & 2 & 2 & 2 & 4 & 3 & 2 & 3 & 1 & 4 & 3 & 3 & 3 & 3 & 3 & 2 & & & & & & \\
\hline & 2 & 2 & 2 & 1 & 3 & 3 & 1 & 2 & 3 & 3 & 2 & 2 & 2 & 3 & 3 & 4 & 2 & 2 & 3 & 4 & 3 & 3 & 3 & 1 & 3 & 4 & 2 & & & & & & \\
\hline & 4 & 3 & 2 & 3 & 2 & 1 & 2 & 3 & 3 & 2 & 2 & 3 & 2 & 2 & 2 & 3 & 3 & 1 & 1 & 3 & 3 & 2 & 3 & 2 & 2 & 2 & 1 & & & & & & \\
\hline 9 & 2 & 2 & 3 & 3 & 3 & 2 & 4 & 2 & 3 & 2 & 3 & 4 & 3 & 3 & 3 & 1 & 2 & 2 & 2 & 2 & 3 & 3 & 3 & 2 & 2 & 2 & 1 & & & & & & \\
\hline & 3 & 2 & 2 & 3 & 2 & 3 & 4 & 3 & 3 & 2 & 3 & 3 & 1 & 3 & 3 & 3 & 2 & 2 & 3 & 1 & 2 & 2 & 2 & 2 & 1 & 2 & 1 & & & & & & \\
\hline 10 & 1 & 4 & 5 & 3 & 4 & 4 & 2 & 2 & 3 & 3 & 3 & 2 & 3 & 3 & 3 & 2 & 3 & 3 & 3 & 3 & 3 & 3 & 2 & 2 & 2 & 2 & 2 & & & & & & \\
\hline & 4 & 2 & 3 & 2 & 3 & 1 & 3 & 3 & 3 & 3 & 4 & 4 & 3 & 3 & 3 & 4 & 4 & 2 & 3 & 5 & 4 & 4 & 2 & 2 & 2 & 2 & 2 & 2 & & & & & \\
\hline & 4 & 4 & 3 & 2 & 3 & 2 & 4 & 5 & 2 & 3 & 3 & 3 & 3 & 3 & 2 & 3 & 3 & 3 & 3 & 3 & 1 & 3 & 2 & 3 & 3 & 3 & 3 & 3 & & & & & \\
\hline 1 & 2 & 1 & 4 & 4 & 4 & 3 & 4 & 3 & 3 & 5 & 3 & 3 & 5 & 3 & 3 & 2 & 3 & 3 & 2 & 2 & 2 & 3 & 2 & 3 & 3 & 3 & 3 & 2 & & & & & \\
\hline & 5 & 2 & 4 & 1 & 3 & 4 & 3 & 3 & 3 & 3 & 3 & 3 & 3 & 2 & 2 & 2 & 4 & 4 & 4 & 4 & 2 & 4 & 4 & 4 & 4 & 2 & 2 & 2 & & & & & \\
\hline & 4 & 2 & 3 & 3 & 4 & 4 & 1 & 2 & 4 & 3 & 2 & 3 & 3 & 3 & 3 & 4 & 3 & 3 & 4 & 4 & 2 & 3 & 2 & 3 & 5 & 5 & 5 & 5 & 4 & & & & \\
\hline & 4 & 3 & 2 & 3 & 3 & 1 & 4 & 3 & 5 & 3 & 5 & 5 & 5 & 4 & 3 & 3 & 4 & 3 & 4 & 3 & 3 & 4 & 3 & 3 & 3 & 3 & 3 & 3 & 3 & & & & \\
\hline 12 & 2 & 4 & 5 & 3 & 3 & 2 & 3 & 2 & 1 & 3 & 3 & 3 & 3 & 2 & 3 & 3 & 2 & 3 & 3 & 3 & 3 & 3 & 3 & 2 & 3 & 2 & 2 & 2 & 2 & & & & \\
\hline & 4 & 3 & 2 & 4 & 3 & 2 & 1 & 4 & 5 & 3 & 3 & 3 & 5 & 3 & 4 & 4 & 4 & 4 & 4 & 4 & 4 & 4 & 4 & 4 & 4 & 4 & 4 & 4 & 4 & & & & \\
\hline & 3 & 3 & 2 & 1 & 2 & 3 & 3 & 5 & 3 & 4 & 4 & 4 & 4 & 3 & 4 & 4 & 3 & 4 & 4 & 4 & 3 & 4 & 3 & 3 & 3 & 3 & 3 & 3 & 3 & & & & \\
\hline & 4 & 4 & 3 & 4 & 3 & 4 & 3 & 4 & 4 & 1 & 5 & 4 & 4 & 5 & 3 & 4 & 5 & 6 & 4 & 2 & 3 & 2 & 4 & 3 & 3 & 3 & 2 & 4 & 2 & 2 & 2 & & \\
\hline 1 & 4 & 3 & 4 & 2 & 4 & 5 & 5 & 6 & 3 & 1 & 1 & 3 & 3 & 3 & 3 & 3 & 3 & 3 & 3 & 2 & 3 & 3 & 3 & 3 & 3 & 3 & 2 & 2 & 2 & 2 & 2 & & \\
\hline & 2 & 4 & 4 & 2 & 5 & 4 & 3 & 1 & 3 & 5 & 4 & 3 & 3 & 3 & 3 & 4 & 3 & 3 & 3 & 4 & 4 & 3 & 3 & 4 & 4 & 3 & 3 & 3 & 3 & 3 & 3 & & \\
\hline & 3 & 2 & 1 & 3 & 3 & 1 & 2 & 3 & 5 & 3 & 4 & 2 & 4 & 4 & 4 & 3 & 3 & 3 & 3 & 3 & 3 & 3 & 3 & 3 & 3 & 3 & 3 & 3 & 3 & 3 & 3 & & \\
\hline 1 & 3 & 3 & 3 & 3 & 3 & 3 & 4 & 2 & 2 & 1 & 4 & 2 & 4 & 5 & 4 & 4 & 3 & 3 & 4 & 3 & 1 & 3 & 4 & 4 & 4 & 3 & 3 & 3 & 4 & 4 & $=4$ & & \\
\hline & 3 & 2 & 3 & 3 & 4 & 5 & 3 & 2 & 5 & 3 & 3 & 3 & 1 & 3 & 2 & 3 & 3 & 2 & 1 & 3 & 3 & 3 & 2 & 2 & 2 & 2 & 2 & 2 & 2 & 2 & 2 & & 2 \\
\hline 15 & 1 & 3 & 3 & 4 & 3 & 5 & 4 & 4 & 2 & 3 & 3 & 3 & 5 & 2 & 6 & 5 & 5 & 4 & 4 & 4 & 4 & 4 & 4 & 2 & 4 & 4 & 4 & 2 & 2 & 2 & 2 & 2 & 2 \\
\hline
\end{tabular}

(e) Best bee out of the first iteration and generation of next iteration bees

The best bee based on evaluating the updated employed and unemployed bees is selected from the first iteration. The same list of employed and unemployed bees concerning the minimum value of assembly specification is selected as the next iteration bees. (f) Best solution: identification

The above steps are repeated till the termination criteria as stated in Table 2 to identify the best solution. Furthermore, the proposed ABC algorithm's sorted list of possible solutions is presented in Tables 9 and 10 for linear assembly. Tables 11-19 represent the best values obtained for KJA with three arms. 
TABLE 16: Best bin combinations for dimension $X_{3 b}$.

\begin{tabular}{|c|c|c|c|c|c|c|c|c|c|c|c|c|c|c|c|c|c|c|c|c|c|c|c|c|c|c|c|c|c|c|c|c|}
\hline Pr. no. & \multicolumn{32}{|c|}{ Best bin combinations } \\
\hline \multirow{2}{*}{1} & 2 & 2 & 3 & 2 & 2 & 2 & 2 & 3 & 2 & 2 & 3 & 1 & 1 & 1 & 1 & 1 & 2 & & & & & & & & & & & & & & & \\
\hline & 2 & 2 & 2 & 3 & 2 & 1 & 3 & 2 & 2 & 1 & 2 & 2 & 2 & 2 & 2 & 2 & 2 & & & & & & & & & & & & & & & \\
\hline \multirow{3}{*}{2} & 2 & 2 & 3 & 2 & 2 & 2 & 1 & 2 & 2 & 1 & 2 & 2 & 2 & 2 & 1 & 1 & 2 & 2 & 2 & 2 & 3 & & & & & & & & & & & \\
\hline & 2 & 2 & 2 & 1 & 2 & 2 & 2 & 3 & 1 & 3 & 3 & 2 & 3 & 2 & 3 & 3 & 2 & 2 & 2 & 2 & 2 & & & & & & & & & & & \\
\hline & 2 & 2 & 1 & 1 & 2 & 2 & 2 & 2 & 2 & 2 & 3 & 3 & 2 & 2 & 2 & 2 & 3 & 2 & 2 & 2 & 2 & & & & & & & & & & & \\
\hline \multirow{4}{*}{3} & 1 & 2 & 3 & 4 & 2 & 4 & 2 & 2 & 3 & 2 & 4 & 3 & 3 & 3 & 3 & 3 & 3 & 3 & 3 & 3 & 3 & 3 & 2 & 3 & & & & & & & & \\
\hline & 3 & 2 & 3 & 2 & 4 & 3 & 1 & 4 & 3 & 1 & 3 & 3 & 3 & 3 & 3 & 3 & 3 & 3 & 3 & 3 & 3 & 3 & 3 & 2 & & & & & & & & \\
\hline & 3 & 2 & 1 & 3 & 3 & 2 & 3 & 4 & 2 & 4 & 2 & 2 & 2 & 2 & 2 & 2 & 2 & 2 & 2 & 2 & 2 & 2 & 3 & 2 & & & & & & & & \\
\hline & 1 & 4 & 3 & 3 & 3 & 3 & 2 & 3 & 2 & 2 & 2 & 2 & 2 & 2 & 2 & 2 & 2 & 2 & 2 & 2 & 2 & 2 & 2 & 3 & & & & & & & & \\
\hline \multirow{4}{*}{4} & 1 & 3 & 3 & 2 & 2 & 2 & 3 & 2 & 3 & 2 & 3 & 3 & 2 & 1 & 4 & 4 & 4 & 4 & 4 & 1 & 1 & 3 & 3 & 1 & 4 & 2 & & & & & & \\
\hline & 4 & 2 & 3 & 3 & 2 & 2 & 3 & 2 & 3 & 3 & 2 & 3 & 2 & 1 & 2 & 1 & 2 & 3 & 3 & 3 & 2 & 1 & 3 & 2 & 1 & 3 & & & & & & \\
\hline & 1 & 3 & 3 & 3 & 2 & 2 & 2 & 3 & 2 & 4 & 2 & 3 & 3 & 4 & 2 & 3 & 3 & 3 & 2 & 3 & 3 & 3 & 2 & 2 & 3 & 2 & & & & & & \\
\hline & 2 & 3 & 3 & 2 & 4 & 3 & 3 & 2 & 3 & 3 & 2 & 2 & 2 & 2 & 2 & 2 & 2 & 2 & 1 & 1 & 1 & 1 & 1 & 2 & 3 & 2 & & & & & & \\
\hline \multirow{3}{*}{5} & 3 & 3 & 2 & 1 & 2 & 4 & 2 & 3 & 2 & 4 & 3 & 3 & 3 & 3 & 3 & 3 & 3 & 3 & 3 & 3 & 3 & 3 & 3 & 3 & 3 & & & & & & & \\
\hline & 3 & 2 & 3 & 2 & 1 & 4 & 2 & 3 & 2 & 3 & 3 & 3 & 3 & 2 & 3 & 3 & 3 & 2 & 3 & 2 & 2 & 2 & 2 & 2 & 2 & & & & & & & \\
\hline & 3 & 3 & 4 & 2 & 4 & 2 & 1 & 2 & 1 & 1 & 2 & 3 & 3 & 3 & 3 & 3 & 4 & 3 & 4 & 4 & 4 & 3 & 3 & 3 & 3 & & & & & & & \\
\hline \multirow{3}{*}{6} & 2 & 3 & 2 & 2 & 1 & 3 & 4 & 3 & 3 & 2 & 4 & 2 & 3 & 3 & 3 & 2 & 2 & 3 & 3 & 3 & 3 & 3 & 3 & 2 & 4 & 2 & & & & & & \\
\hline & 3 & 1 & 3 & 2 & 3 & 2 & 4 & 3 & 2 & 2 & 4 & 2 & 2 & 2 & 2 & 3 & 2 & 3 & 3 & 4 & 2 & 2 & 2 & 2 & 3 & 1 & & & & & & \\
\hline & 3 & 2 & 3 & 3 & 2 & 2 & 3 & 1 & 4 & 2 & 4 & 2 & 4 & 2 & 2 & 2 & 2 & 2 & 2 & 2 & 2 & 2 & 2 & 2 & 3 & 2 & & & & & & \\
\hline \multirow{4}{*}{7} & 3 & 4 & 1 & 3 & 3 & 2 & 2 & 4 & 3 & 2 & 3 & 2 & 3 & 2 & 3 & 3 & 3 & 3 & 3 & 3 & 3 & 3 & 3 & 3 & 3 & 3 & & & & & & \\
\hline & 1 & 4 & 3 & 3 & 3 & 2 & 2 & 2 & 2 & 2 & 3 & 2 & 3 & 3 & 2 & 3 & 3 & 3 & 3 & 3 & 3 & 3 & 3 & 3 & 3 & 3 & & & & & & \\
\hline & 3 & 3 & 1 & 4 & 3 & 1 & 2 & 3 & 2 & 2 & 2 & 2 & 2 & 2 & 2 & 2 & 2 & 2 & 2 & 2 & 2 & 2 & 2 & 2 & 2 & 2 & & & & & & \\
\hline & 2 & 3 & 4 & 2 & 1 & 3 & 2 & 3 & 3 & 3 & 2 & 3 & 4 & 2 & 2 & 2 & 2 & 2 & 2 & 2 & 2 & 2 & 2 & 2 & 2 & 3 & & & & & & \\
\hline & 2 & 2 & 3 & 4 & 2 & 1 & 4 & 3 & 3 & 3 & 3 & 3 & 3 & 3 & 3 & 3 & 3 & 3 & 3 & 3 & 3 & 3 & 3 & 3 & 3 & 3 & 2 & & & & & \\
\hline 8 & 2 & 3 & 2 & 3 & 3 & 2 & 2 & 2 & 1 & 2 & 3 & 2 & 4 & 4 & 4 & 2 & 2 & 2 & 2 & 2 & 2 & 2 & 2 & 2 & 2 & 2 & 3 & & & & & \\
\hline & 2 & 3 & 4 & 1 & 3 & 2 & 1 & 4 & 3 & 4 & 2 & 3 & 2 & 2 & 4 & 3 & 3 & 3 & 4 & 3 & 3 & 3 & 3 & 3 & 3 & 3 & 3 & & & & & \\
\hline & 3 & 3 & 2 & 1 & 3 & 5 & 3 & 5 & 3 & 3 & 3 & 4 & 3 & 3 & 4 & 3 & 4 & 4 & 4 & 4 & 4 & 4 & 4 & 4 & 4 & 4 & 4 & & & & & \\
\hline 9 & 3 & 3 & 4 & 1 & 3 & 4 & 2 & 3 & 5 & 3 & 4 & 4 & 3 & 3 & 3 & 4 & 4 & 2 & 2 & 2 & 2 & 2 & 2 & 2 & 2 & 2 & 4 & & & & & \\
\hline & 4 & 4 & 4 & 5 & 3 & 2 & 3 & 3 & 3 & 3 & 3 & 3 & 3 & 3 & 1 & 3 & 3 & 3 & 3 & 3 & 3 & 3 & 3 & 1 & 3 & 3 & 3 & & & & & \\
\hline 10 & 3 & 2 & 3 & 4 & 4 & 3 & 2 & 4 & 1 & 3 & 3 & 4 & 3 & 3 & 3 & 5 & 5 & 5 & 4 & 4 & 2 & 2 & 2 & 2 & 2 & 2 & 2 & & & & & \\
\hline & 3 & 4 & 3 & 4 & 2 & 3 & 3 & 3 & 4 & 4 & 3 & 2 & 3 & 1 & 4 & 5 & 5 & 2 & 3 & 3 & 3 & 3 & 3 & 3 & 3 & 3 & 3 & 3 & & & & \\
\hline & 2 & 4 & 4 & 4 & 5 & 3 & 3 & 3 & 2 & 4 & 3 & 3 & 5 & 3 & 3 & 3 & 3 & 5 & 1 & 3 & 3 & 3 & 3 & 3 & 3 & 3 & 3 & 3 & & & & \\
\hline 1 & 4 & 3 & 4 & 1 & 5 & 3 & 3 & 3 & 3 & 3 & 3 & 3 & 3 & 2 & 3 & 3 & 3 & 2 & 3 & 3 & 2 & 3 & 2 & 2 & 2 & 2 & 2 & 2 & & & & \\
\hline & 4 & 3 & 3 & 3 & 2 & 2 & 1 & 3 & 3 & 3 & 4 & 5 & 4 & 2 & 4 & 4 & 4 & 4 & 4 & 4 & 2 & 4 & 2 & 4 & 4 & 4 & 4 & 4 & & & & \\
\hline & 3 & 4 & 3 & 3 & 2 & 1 & 5 & 5 & 4 & 2 & 4 & 3 & 3 & 3 & 4 & 4 & 3 & 4 & 4 & 3 & 3 & 3 & 3 & 3 & 3 & 3 & 3 & 3 & 3 & & & \\
\hline & 2 & 3 & 4 & 3 & 5 & 4 & 3 & 4 & 4 & 1 & 2 & 3 & 3 & 2 & 5 & 3 & 3 & 3 & 3 & 3 & 3 & 3 & 2 & 3 & 3 & 3 & 2 & 2 & 2 & & & \\
\hline 12 & 4 & 3 & 3 & 2 & 3 & 5 & 2 & 2 & 4 & 1 & 4 & 4 & 1 & 2 & 4 & 3 & 5 & 3 & 3 & 2 & 3 & 3 & 3 & 4 & 4 & 4 & 4 & 2 & 2 & & & \\
\hline & 4 & 3 & 2 & 3 & 4 & 4 & 3 & 5 & 1 & 3 & 3 & 5 & 3 & 3 & 3 & 5 & 3 & 4 & 4 & 4 & 4 & 4 & 4 & 4 & 4 & 4 & 4 & 4 & 4 & & & \\
\hline & 3 & 3 & 3 & 4 & 3 & 2 & 3 & 3 & 5 & 3 & 2 & 1 & 5 & 4 & 4 & 4 & 4 & 4 & 2 & 2 & 4 & 4 & 4 & 4 & 4 & 2 & 2 & 2 & 2 & & & \\
\hline & 3 & 4 & 4 & 4 & 3 & 3 & 3 & 3 & 4 & 3 & 5 & 3 & 2 & 5 & 4 & 5 & 4 & 1 & 4 & 5 & 4 & 5 & 6 & 6 & 5 & 4 & 2 & 4 & 4 & 4 & 4 & 3 \\
\hline 13 & 5 & 6 & 3 & 2 & 4 & 3 & 3 & 4 & 4 & 3 & 3 & 3 & 4 & 4 & 4 & 3 & 1 & 4 & 3 & 4 & 2 & 4 & 4 & 3 & 3 & 3 & 3 & 2 & 2 & 2 & 2 & 2 \\
\hline & 4 & 3 & 4 & 4 & 3 & 5 & 4 & 3 & 2 & 3 & 1 & 6 & 5 & 4 & 4 & 3 & 4 & 3 & 3 & 6 & 6 & 5 & 3 & 3 & 3 & 3 & 3 & 3 & 3 & 3 & 3 & 3 \\
\hline & 1 & 3 & 5 & 6 & 3 & 3 & 3 & 4 & 5 & 4 & 4 & 3 & 2 & 4 & 4 & 4 & 3 & 2 & 3 & 5 & 4 & 3 & 3 & 5 & 3 & 3 & 5 & 2 & 2 & 2 & 2 & 2 \\
\hline 14 & 4 & 2 & 4 & 3 & 4 & 5 & 5 & 3 & 4 & 2 & 4 & 3 & 2 & 3 & 4 & 5 & 1 & 6 & 3 & 3 & 3 & 3 & 3 & 3 & 3 & 3 & 3 & 3 & 3 & 3 & 2 & 1 \\
\hline & 5 & 4 & 3 & 3 & 4 & 5 & 4 & 4 & 3 & 3 & 3 & 3 & 3 & 1 & 4 & 2 & 6 & 4 & 3 & 5 & 4 & 3 & 4 & 3 & 3 & 3 & 3 & 3 & 3 & 3 & 3 & 1 \\
\hline 15 & 3 & 4 & 1 & 4 & 4 & 3 & 3 & 2 & 4 & 4 & 5 & 3 & 2 & 6 & 4 & 5 & 5 & 2 & 5 & 4 & 4 & 6 & 3 & 3 & 3 & 3 & 3 & 3 & 3 & 3 & 2 & \\
\hline
\end{tabular}

\section{Results and Discussion}

The new grouping method is applied to minimize the manufacturing cost of both linear and complex assemblies. In linear assembly, compared with the existing method, the length of the bin combination is reduced by introducing a new method. The proposed method has arrived at a clearance variation of $15 \mu \mathrm{m}$, which is $2.5 \mu \mathrm{m}$ less than the existing method dealt by Wang et al. [33]. Tables 9, 10, and 20 represent the best combination of bins, $C V$, and $N A k$ for 
TABLE 17: Best bin combinations for dimension $X_{4}$.

\begin{tabular}{|c|c|c|c|c|c|c|c|c|c|c|c|c|c|c|c|c|c|c|c|c|c|c|c|c|c|c|c|c|c|c|c|c|}
\hline Pr. no. & \multicolumn{32}{|c|}{ Best bin combinations } \\
\hline \multirow{2}{*}{1} & 4 & 2 & 1 & 3 & 2 & 3 & 3 & 3 & 2 & 2 & 2 & 3 & 2 & 2 & 2 & 2 & 3 & & & & & & & & & & & & & & & \\
\hline & 2 & 2 & 3 & 3 & 1 & 2 & 2 & 2 & 1 & 4 & 3 & 3 & 3 & 3 & 4 & 4 & 4 & & & & & & & & & & & & & & & \\
\hline \multirow{3}{*}{2} & 1 & 2 & 2 & 3 & 3 & 2 & 4 & 2 & 3 & 3 & 2 & 2 & 2 & 2 & 2 & 2 & 3 & 3 & 1 & 2 & 2 & & & & & & & & & & & \\
\hline & 3 & 1 & 2 & 2 & 3 & 3 & 4 & 2 & 2 & 2 & 2 & 2 & 2 & 2 & 2 & 2 & 3 & 3 & 1 & 1 & 2 & & & & & & & & & & & \\
\hline & 1 & 4 & 2 & 3 & 3 & 2 & 3 & 1 & 3 & 3 & 2 & 2 & 2 & 3 & 3 & 3 & 1 & 2 & 3 & 2 & 2 & & & & & & & & & & & \\
\hline \multirow{4}{*}{3} & 3 & 3 & 4 & 1 & 2 & 2 & 5 & 3 & 4 & 4 & 2 & 2 & 4 & 4 & 3 & 2 & 3 & 3 & 3 & 4 & 4 & 4 & 3 & 2 & & & & & & & & \\
\hline & 3 & 3 & 3 & 4 & 1 & 3 & 5 & 2 & 4 & 4 & 4 & 4 & 2 & 4 & 4 & 2 & 4 & 2 & 2 & 1 & 2 & 2 & 2 & 3 & & & & & & & & \\
\hline & 5 & 1 & 2 & 2 & 3 & 4 & 3 & 2 & 3 & 4 & 4 & 3 & 4 & 4 & 2 & 4 & 2 & 2 & 2 & 2 & 2 & 2 & 2 & 3 & & & & & & & & \\
\hline & 3 & 3 & 4 & 5 & 2 & 4 & 4 & 3 & 4 & 3 & 3 & 3 & 3 & 1 & 3 & 3 & 3 & 3 & 3 & 3 & 3 & 1 & 2 & 1 & & & & & & & & \\
\hline \multirow{4}{*}{4} & 5 & 2 & 2 & 3 & 4 & 1 & 3 & 2 & 3 & 2 & 2 & 3 & 2 & 3 & 3 & 2 & 2 & 2 & 2 & 2 & 2 & 3 & 3 & 4 & 3 & 1 & & & & & & \\
\hline & 2 & 4 & 2 & 3 & 5 & 2 & 3 & 3 & 3 & 3 & 3 & 3 & 1 & 3 & 3 & 3 & 2 & 2 & 2 & 2 & 2 & 2 & 3 & 2 & 3 & 2 & & & & & & \\
\hline & 2 & 3 & 2 & 3 & 2 & 5 & 3 & 4 & 3 & 3 & 4 & 4 & 4 & 3 & 3 & 4 & 1 & 3 & 3 & 3 & 3 & 3 & 2 & 2 & 3 & 3 & & & & & & \\
\hline & 3 & 3 & 2 & 4 & 2 & 5 & 4 & 2 & 3 & 2 & 2 & 1 & 3 & 3 & 3 & 3 & 3 & 3 & 3 & 3 & 2 & 3 & 2 & 4 & 1 & 3 & & & & & & \\
\hline \multirow{3}{*}{5} & 1 & 2 & 2 & 4 & 2 & 2 & 3 & 3 & 3 & 3 & 3 & 3 & 2 & 3 & 3 & 3 & 3 & 3 & 3 & 3 & 3 & 3 & 3 & 3 & 3 & & & & & & & \\
\hline & 2 & 1 & 3 & 3 & 3 & 2 & 4 & 3 & 1 & 3 & 3 & 3 & 2 & 3 & 3 & 2 & 2 & 2 & 2 & 2 & 2 & 2 & 2 & 2 & 2 & & & & & & & \\
\hline & 1 & 4 & 3 & 3 & 4 & 2 & 2 & 3 & 2 & 4 & 2 & 3 & 3 & 2 & 2 & 4 & 2 & 2 & 2 & 2 & 2 & 2 & 2 & 2 & 2 & & & & & & & \\
\hline \multirow{3}{*}{6} & 1 & 2 & 2 & 2 & 4 & 2 & 2 & 3 & 2 & 3 & 3 & 3 & 3 & 3 & 3 & 3 & 3 & 3 & 3 & 3 & 3 & 3 & 3 & 2 & 1 & 3 & & & & & & \\
\hline & 3 & 3 & 2 & 2 & 2 & 1 & 2 & 1 & 3 & 4 & 3 & 2 & 2 & 2 & 3 & 3 & 2 & 3 & 3 & 3 & 3 & 3 & 3 & 3 & 2 & 2 & & & & & & \\
\hline & 3 & 4 & 2 & 3 & 1 & 3 & 3 & 3 & 2 & 2 & 2 & 2 & 2 & 1 & 4 & 2 & 3 & 2 & 3 & 2 & 3 & 4 & 2 & 2 & 1 & 2 & & & & & & \\
\hline \multirow{4}{*}{7} & 3 & 2 & 3 & 1 & 4 & 2 & 2 & 2 & 3 & 3 & 3 & 2 & 2 & 2 & 3 & 3 & 3 & 2 & 3 & 3 & 3 & 2 & 3 & 3 & 3 & 3 & & & & & & \\
\hline & 2 & 2 & 1 & 2 & 2 & 4 & 3 & 3 & 4 & 3 & 2 & 2 & 3 & 3 & 3 & 2 & 3 & 3 & 3 & 3 & 3 & 3 & 3 & 3 & 3 & 3 & & & & & & \\
\hline & 2 & 3 & 3 & 1 & 3 & 3 & 4 & 3 & 3 & 3 & 4 & 2 & 3 & 2 & 3 & 3 & 3 & 3 & 3 & 1 & 3 & 3 & 3 & 3 & 3 & 3 & & & & & & \\
\hline & 3 & 2 & 3 & 2 & 4 & 3 & 2 & 1 & 3 & 3 & 3 & 3 & 3 & 3 & 3 & 3 & 3 & 3 & 3 & 3 & 3 & 3 & 3 & 3 & 3 & 3 & & & & & & \\
\hline & 2 & 2 & 3 & 3 & 2 & 3 & 2 & 4 & 3 & 1 & 3 & 1 & 3 & 2 & 3 & 3 & 3 & 3 & 3 & 3 & 3 & 3 & 3 & 3 & 3 & 3 & 3 & & & & & \\
\hline 8 & 2 & 3 & 3 & 3 & 1 & 3 & 1 & 4 & 2 & 2 & 2 & 2 & 2 & 2 & 2 & 2 & 2 & 2 & 2 & 2 & 2 & 2 & 2 & 2 & 2 & 2 & 2 & & & & & \\
\hline & 3 & 3 & 1 & 3 & 2 & 3 & 2 & 3 & 1 & 2 & 2 & 4 & 4 & 2 & 3 & 3 & 3 & 2 & 2 & 2 & 2 & 2 & 2 & 2 & 2 & 2 & 3 & & & & & \\
\hline & 2 & 1 & 2 & 2 & 2 & 2 & 3 & 3 & 3 & 1 & 3 & 3 & 4 & 2 & 3 & 4 & 3 & 4 & 2 & 2 & 2 & 2 & 2 & 2 & 2 & 2 & 2 & & & & & \\
\hline 9 & 2 & 3 & 2 & 2 & 1 & 3 & 4 & 3 & 2 & 2 & 2 & 3 & 3 & 2 & 3 & 3 & 3 & 2 & 3 & 3 & 2 & 3 & 2 & 2 & 2 & 2 & 3 & & & & & \\
\hline & 2 & 1 & 2 & 2 & 3 & 2 & 3 & 3 & 2 & 3 & 3 & 2 & 2 & 4 & 4 & 4 & 3 & 3 & 3 & 3 & 3 & 3 & 3 & 3 & 3 & 3 & 3 & & & & & \\
\hline 10 & 5 & 3 & 4 & 4 & 1 & 3 & 1 & 2 & 3 & 2 & 4 & 4 & 6 & 4 & 5 & 3 & 4 & 3 & 5 & 3 & 3 & 3 & 5 & 5 & 4 & 3 & 4 & & & & & \\
\hline & 4 & 4 & 4 & 4 & 2 & 5 & 6 & 3 & 3 & 5 & 3 & 5 & 3 & 5 & 3 & 3 & 3 & 3 & 3 & 3 & 3 & 3 & 1 & 3 & 3 & 3 & 3 & 3 & & & & \\
\hline & 3 & 4 & 3 & 5 & 5 & 4 & 6 & 2 & 4 & 4 & 3 & 2 & 6 & 4 & 5 & 5 & 3 & 4 & 4 & 3 & 3 & 3 & 3 & 3 & 1 & 3 & 4 & 3 & & & & \\
\hline 1 & 3 & 4 & 4 & 5 & 4 & 3 & 4 & 2 & 5 & 4 & 1 & 3 & 4 & 4 & 5 & 3 & 4 & 2 & 3 & 6 & 3 & 3 & 3 & 3 & 2 & 3 & 2 & 3 & & & & \\
\hline & 3 & 5 & 5 & 4 & 4 & 2 & 4 & 3 & 3 & 4 & 6 & 4 & 3 & 3 & 1 & 2 & 4 & 4 & 4 & 4 & 4 & 4 & 2 & 4 & 4 & 4 & 2 & 2 & & & & \\
\hline & 3 & 5 & 6 & 4 & 4 & 4 & 4 & 3 & 3 & 4 & 1 & 4 & 3 & 4 & 3 & 2 & 3 & 3 & 3 & 2 & 3 & 3 & 2 & 2 & 2 & 2 & 2 & 2 & 2 & & & \\
\hline & 3 & 3 & 4 & 5 & 4 & 5 & 4 & 4 & 4 & 3 & 4 & 4 & 3 & 5 & 2 & 2 & 6 & 1 & 5 & 2 & 3 & 3 & 3 & 3 & 3 & 3 & 3 & 3 & 3 & & & \\
\hline 12 & 3 & 3 & 4 & 3 & 5 & 3 & 4 & 4 & 4 & 6 & 5 & 1 & 2 & 6 & 5 & 4 & 2 & 4 & 4 & 4 & 2 & 4 & 4 & 4 & 2 & 2 & 2 & 2 & 2 & & & \\
\hline & 3 & 5 & 4 & 6 & 3 & 3 & 4 & 1 & 2 & 2 & 4 & 3 & 3 & 3 & 3 & 3 & 3 & 3 & 3 & 2 & 3 & 3 & 3 & 2 & 2 & 2 & 2 & 2 & 2 & & & \\
\hline & 4 & 5 & 3 & 3 & 3 & 3 & 2 & 4 & 3 & 4 & 6 & 4 & 3 & 3 & 5 & 5 & 4 & 1 & 4 & 4 & 4 & 4 & 4 & 4 & 4 & 4 & 4 & 4 & 4 & & & \\
\hline 1 & 1 & 3 & 3 & 4 & 3 & 3 & 2 & 3 & 3 & 3 & 3 & 2 & 2 & 2 & 2 & 2 & 2 & 2 & 2 & 2 & 2 & 2 & 2 & 2 & 2 & 2 & 2 & 2 & 2 & 2 & 2 & 2 \\
\hline 1 & 2 & 2 & 1 & 4 & 3 & 3 & 2 & 2 & 3 & 3 & 2 & 2 & 3 & 3 & 3 & 3 & 1 & 2 & 2 & 1 & 3 & 1 & 2 & 2 & 3 & 2 & 2 & 1 & 2 & 2 & 2 & 2 \\
\hline & 2 & 2 & 3 & 3 & 3 & 1 & 3 & 4 & 3 & 2 & 2 & 2 & 2 & 3 & 3 & 4 & 3 & 2 & 2 & 3 & 3 & 2 & 3 & 2 & 3 & 3 & 3 & 2 & 2 & 3 & 3 & 3 \\
\hline & 2 & 2 & 2 & 4 & 3 & 3 & 1 & 4 & 2 & 3 & 3 & 2 & 3 & 3 & 2 & 3 & 2 & 3 & 2 & 2 & 3 & 3 & 3 & 2 & 2 & 2 & 2 & 3 & 3 & 3 & 3 & 3 \\
\hline 14 & 2 & 3 & 2 & 3 & 2 & 1 & 4 & 3 & 2 & 4 & 3 & 2 & 3 & 2 & 2 & 3 & 3 & 3 & 2 & 3 & 3 & 2 & 3 & 3 & 3 & 3 & 3 & 3 & 3 & 3 & 3 & 3 \\
\hline & 2 & 2 & 1 & 1 & 2 & 3 & 4 & 2 & 3 & 3 & 2 & 2 & 4 & 2 & 2 & 3 & 3 & 2 & 3 & 3 & 3 & 3 & 3 & 3 & 3 & 3 & 3 & 3 & 3 & 3 & 3 & 3 \\
\hline 15 & 2 & 3 & 1 & 3 & 3 & 1 & 3 & 4 & 3 & 2 & 2 & 2 & 2 & 3 & 2 & 2 & 2 & 2 & 2 & 2 & 2 & 2 & 2 & 2 & 2 & 2 & 2 & 2 & 2 & 2 & 2 & 1 \\
\hline
\end{tabular}

various bin numbers from 5 to 8 . In Tables 9 and 11, the bolded value of zero indicates that the length of the bin combination can be reduced, and the improved length of the bin is shown in Table 21. Figure 4 represents the dimensional distribution of gears $\mathrm{A}, \mathrm{B}$, and $\mathrm{C}$ according to bin numbers from 3 to 8 . Tables 22 and 23 represent the dimensional distribution/number of parts in each bin for all components of KJA with three arms. Figure 5 illustrates the tolerance of the components both in the existing and proposed method. 
TABLE 18: Best bin combinations for dimension $X_{5}$.

\begin{tabular}{|c|c|c|c|c|c|c|c|c|c|c|c|c|c|c|c|c|c|c|c|c|c|c|c|c|c|c|c|c|c|c|c|c|}
\hline Pr. no. & \multicolumn{32}{|c|}{ Best bin combinations } \\
\hline \multirow{2}{*}{1} & 1 & 2 & 2 & 3 & 3 & 2 & 2 & 2 & 2 & 2 & 2 & 2 & 1 & 2 & 2 & 2 & 2 & & & & & & & & & & & & & & & \\
\hline & 2 & 3 & 2 & 2 & 3 & 3 & 2 & 1 & 2 & 1 & 2 & 2 & 2 & 2 & 2 & 1 & 2 & & & & & & & & & & & & & & & \\
\hline \multirow{3}{*}{2} & 3 & 1 & 2 & 2 & 2 & 2 & 3 & 2 & 2 & 2 & 2 & 3 & 3 & 3 & 3 & 3 & 2 & 3 & 2 & 2 & 1 & & & & & & & & & & & \\
\hline & 3 & 2 & 2 & 2 & 2 & 2 & 2 & 2 & 2 & 2 & 2 & 2 & 2 & 1 & 1 & 1 & 2 & 2 & 2 & 2 & 2 & & & & & & & & & & & \\
\hline & 2 & 2 & 3 & 2 & 1 & 2 & 3 & 2 & 2 & 2 & 2 & 2 & 2 & 3 & 2 & 3 & 2 & 3 & 2 & 3 & 2 & & & & & & & & & & & \\
\hline \multirow{4}{*}{3} & 1 & 3 & 2 & 2 & 2 & 2 & 2 & 2 & 2 & 1 & 2 & 2 & 2 & 2 & 2 & 2 & 1 & 2 & 2 & 2 & 2 & 2 & 2 & 3 & & & & & & & & \\
\hline & 2 & 2 & 3 & 2 & 2 & 3 & 2 & 3 & 1 & 2 & 1 & 1 & 2 & 2 & 2 & 2 & 3 & 3 & 1 & 1 & 1 & 1 & 2 & 2 & & & & & & & & \\
\hline & 2 & 2 & 2 & 3 & 2 & 2 & 2 & 1 & 2 & 2 & 2 & 2 & 3 & 3 & 2 & 3 & 3 & 3 & 3 & 3 & 3 & 3 & 3 & 1 & & & & & & & & \\
\hline & 3 & 2 & 1 & 2 & 2 & 2 & 2 & 2 & 2 & 3 & 3 & 3 & 2 & 2 & 2 & 3 & 2 & 3 & 3 & 3 & 2 & 2 & 2 & 2 & & & & & & & & \\
\hline \multirow{4}{*}{4} & 2 & 1 & 2 & 3 & 2 & 2 & 3 & 2 & 2 & 2 & 3 & 2 & 2 & 2 & 2 & 2 & 2 & 2 & 2 & 2 & 2 & 2 & 2 & 2 & 2 & 2 & & & & & & \\
\hline & 3 & 2 & 2 & 2 & 2 & 2 & 3 & 2 & 2 & 1 & 3 & 2 & 3 & 2 & 2 & 3 & 3 & 3 & 3 & 3 & 3 & 3 & 3 & 2 & 2 & 3 & & & & & & \\
\hline & 2 & 2 & 3 & 1 & 3 & 3 & 3 & 2 & 2 & 2 & 2 & 2 & 2 & 2 & 2 & 2 & 2 & 2 & 2 & 2 & 2 & 2 & 2 & 2 & 2 & 1 & & & & & & \\
\hline & 2 & 1 & 3 & 2 & 3 & 2 & 2 & 2 & 2 & 2 & 3 & 3 & 2 & 2 & 3 & 2 & 3 & 2 & 2 & 2 & 2 & 2 & 2 & 1 & 3 & 2 & & & & & & \\
\hline \multirow{3}{*}{5} & 4 & 1 & 4 & 5 & 3 & 4 & 4 & 2 & 2 & 3 & 5 & 3 & 4 & 2 & 3 & 4 & 3 & 4 & 4 & 3 & 3 & 3 & 2 & 2 & 3 & & & & & & & \\
\hline & 3 & 3 & 1 & 3 & 3 & 2 & 4 & 5 & 3 & 5 & 3 & 2 & 4 & 5 & 4 & 4 & 2 & 4 & 4 & 4 & 4 & 4 & 4 & 4 & 4 & & & & & & & \\
\hline & 4 & 1 & 4 & 3 & 4 & 3 & 3 & 5 & 5 & 5 & 5 & 5 & 4 & 2 & 4 & 4 & 4 & 4 & 4 & 2 & 4 & 4 & 2 & 4 & 4 & & & & & & & \\
\hline \multirow{3}{*}{6} & 4 & 3 & 3 & 4 & 4 & 4 & 4 & 4 & 4 & 4 & 5 & 6 & 5 & 5 & 5 & 5 & 5 & 5 & 2 & 2 & 1 & 2 & 2 & 5 & 2 & 5 & & & & & & \\
\hline & 3 & 3 & 5 & 4 & 3 & 5 & 6 & 4 & 2 & 4 & 4 & 4 & 4 & 3 & 1 & 4 & 4 & 4 & 3 & 3 & 4 & 4 & 4 & 3 & 6 & 4 & & & & & & \\
\hline & 4 & 4 & 5 & 3 & 3 & 1 & 2 & 4 & 6 & 3 & 3 & 4 & 4 & 4 & 5 & 3 & 2 & 4 & 5 & 5 & 3 & 3 & 3 & 1 & 5 & 5 & & & & & & \\
\hline \multirow{4}{*}{7} & 5 & 4 & 3 & 4 & 5 & 1 & 3 & 4 & 4 & 3 & 4 & 2 & 3 & 4 & 4 & 6 & 5 & 5 & 5 & 5 & 5 & 5 & 5 & 5 & 5 & 5 & & & & & & \\
\hline & 5 & 5 & 3 & 3 & 4 & 5 & 5 & 4 & 4 & 6 & 5 & 4 & 1 & 4 & 4 & 4 & 4 & 4 & 4 & 4 & 2 & 4 & 4 & 2 & 2 & 2 & & & & & & \\
\hline & 4 & 3 & 4 & 5 & 2 & 3 & 4 & 4 & 4 & 1 & 3 & 6 & 5 & 5 & 3 & 5 & 5 & 2 & 3 & 3 & 3 & 3 & 3 & 3 & 2 & 1 & & & & & & \\
\hline & 6 & 3 & 5 & 4 & 4 & 3 & 4 & 5 & 4 & 5 & 4 & 2 & 5 & 5 & 5 & 1 & 4 & 4 & 2 & 4 & 4 & 4 & 4 & 2 & 2 & 4 & & & & & & \\
\hline & 5 & 5 & 4 & 4 & 4 & 4 & 6 & 2 & 3 & 3 & 4 & 3 & 4 & 3 & 3 & 6 & 1 & 4 & 3 & 3 & 3 & 2 & 3 & 3 & 3 & 3 & 1 & & & & & \\
\hline 8 & 5 & 4 & 5 & 3 & 4 & 4 & 1 & 2 & 5 & 4 & 4 & 5 & 4 & 6 & 6 & 6 & 3 & 3 & 3 & 3 & 3 & 2 & 3 & 3 & 1 & 1 & 1 & & & & & \\
\hline & 3 & 3 & 6 & 4 & 5 & 4 & 5 & 4 & 4 & 5 & 5 & 2 & 4 & 4 & 1 & 2 & 4 & 4 & 4 & 4 & 4 & 4 & 2 & 2 & 2 & 2 & 5 & & & & & \\
\hline & 4 & 3 & 5 & 4 & 5 & 1 & 6 & 4 & 3 & 4 & 4 & 4 & 4 & 4 & 4 & 4 & 4 & 4 & 4 & 4 & 4 & 2 & 4 & 4 & 2 & 2 & 2 & & & & & \\
\hline 9 & 5 & 2 & 6 & 4 & 5 & 3 & 4 & 3 & 4 & 4 & 5 & 4 & 3 & 4 & 3 & 4 & 4 & 3 & 4 & 1 & 4 & 4 & 4 & 4 & 4 & 3 & 5 & & & & & \\
\hline & 5 & 4 & 4 & 3 & 2 & 3 & 4 & 6 & 1 & 4 & 4 & 4 & 4 & 5 & 4 & 4 & 5 & 5 & 3 & 3 & 4 & 5 & 4 & 5 & 5 & 5 & 5 & & & & & \\
\hline 10 & 2 & 4 & 2 & 3 & 1 & 3 & 3 & 3 & 3 & 3 & 3 & 3 & 3 & 3 & 2 & 3 & 3 & 3 & 2 & 2 & 2 & 2 & 2 & 2 & 2 & 2 & 2 & & & & & \\
\hline & 4 & 3 & 2 & 3 & 3 & 3 & 3 & 3 & 2 & 2 & 3 & 3 & 3 & 3 & 3 & 1 & 2 & 3 & 3 & 2 & 3 & 2 & 2 & 3 & 2 & 3 & 3 & 3 & & & & \\
\hline & 3 & 3 & 3 & 4 & 3 & 3 & 3 & 2 & 3 & 2 & 1 & 3 & 2 & 3 & 3 & 4 & 4 & 2 & 2 & 2 & 2 & 2 & 2 & 2 & 2 & 2 & 2 & 2 & & & & \\
\hline 1 & 3 & 2 & 3 & 3 & 2 & 3 & 2 & 3 & 1 & 3 & 4 & 4 & 3 & 3 & 3 & 3 & 2 & 2 & 3 & 2 & 3 & 4 & 4 & 4 & 2 & 2 & 2 & 2 & & & & \\
\hline & 3 & 2 & 2 & 2 & 3 & 2 & 3 & 3 & 3 & 2 & 3 & 4 & 3 & 3 & 4 & 2 & 3 & 3 & 3 & 3 & 4 & 3 & 3 & 3 & 3 & 1 & 3 & 1 & & & & \\
\hline & 3 & 3 & 3 & 3 & 1 & 2 & 3 & 2 & 3 & 2 & 2 & 3 & 4 & 3 & 3 & 3 & 4 & 4 & 3 & 2 & 3 & 3 & 3 & 3 & 3 & 2 & 2 & 2 & 2 & & & \\
\hline & 3 & 3 & 2 & 2 & 1 & 3 & 2 & 4 & 3 & 3 & 4 & 3 & 3 & 4 & 3 & 3 & 3 & 3 & 3 & 3 & 3 & 3 & 3 & 3 & 3 & 3 & 3 & 3 & 3 & & & \\
\hline 12 & 4 & 3 & 3 & 3 & 3 & 3 & 2 & 3 & 3 & 3 & 3 & 2 & 3 & 2 & 1 & 3 & 3 & 2 & 2 & 3 & 2 & 2 & 2 & 2 & 2 & 1 & 2 & 2 & 2 & & & \\
\hline & 4 & 2 & 3 & 3 & 3 & 2 & 3 & 3 & 3 & 3 & 3 & 2 & 2 & 2 & 3 & 4 & 2 & 2 & 3 & 1 & 1 & 3 & 4 & 3 & 4 & 4 & 4 & 1 & 1 & & & \\
\hline & 1 & 3 & 3 & 3 & 3 & 3 & 3 & 2 & 3 & 3 & 2 & 2 & 3 & 4 & 3 & 3 & 4 & 2 & 2 & 3 & 4 & 2 & 3 & 2 & 2 & 2 & 2 & 2 & 2 & & & \\
\hline & 5 & 7 & 4 & 5 & 5 & 2 & 6 & 3 & 4 & 3 & 4 & 5 & 5 & 3 & 5 & 6 & 4 & 4 & 4 & 4 & 4 & 4 & 1 & 3 & 6 & 6 & 6 & 6 & 6 & 3 & 3 & \\
\hline 13 & 3 & 4 & 4 & 4 & 4 & 2 & 5 & 6 & 4 & 7 & 5 & 5 & 5 & 5 & 7 & 5 & 6 & 6 & 6 & 6 & 6 & 6 & 5 & 6 & 5 & 5 & 5 & 5 & 5 & 2 & 1 & 1 \\
\hline & 3 & 6 & 5 & 4 & 4 & 4 & 5 & 5 & 5 & 4 & 4 & 1 & 5 & 6 & 7 & 2 & 5 & 4 & 5 & 5 & 4 & 5 & 5 & 5 & 5 & 5 & 4 & 4 & 4 & 4 & 4 & 2 \\
\hline 1 & 5 & 6 & 2 & 3 & 4 & 5 & 5 & 4 & 4 & 1 & 4 & 4 & 4 & 4 & 5 & 3 & 3 & 6 & 4 & 7 & 5 & 3 & 5 & 5 & 5 & 5 & 5 & 5 & 5 & 5 & 3 & 3 \\
\hline 1 & 4 & 3 & 4 & 4 & 5 & 5 & 5 & 5 & 4 & 7 & 1 & 5 & 6 & 3 & 7 & 5 & 7 & 3 & 5 & 6 & 3 & 6 & 6 & 6 & 3 & 2 & 3 & 3 & 3 & 3 & 2 & 2 \\
\hline & 6 & 4 & 4 & 5 & 4 & 3 & 5 & 7 & 3 & 3 & 4 & 5 & 3 & 5 & 5 & 5 & 3 & 5 & 5 & 5 & 5 & 5 & 5 & 5 & 5 & 3 & 2 & 3 & 1 & 3 & 3 & 3 \\
\hline 15 & 4 & 5 & 4 & 1 & 4 & 6 & 3 & 6 & 5 & 5 & 4 & 7 & 5 & 5 & 5 & 4 & 5 & 6 & 5 & 4 & 5 & 4 & 4 & 5 & 5 & 5 & 5 & 2 & 5 & 5 & 1 & 4 \\
\hline
\end{tabular}

The \% of cost savings in each component of KJA with three arms is calculated using the following equation. These values are shown in Figure 6.

$$
\% S_{i}=100 * \frac{\left(M C_{i a}-M C_{i}\right)}{M C_{i a}} .
$$

For example, the $\%$ of cost saving of component $X_{i}$ can be calculated as

$$
\% S_{X_{1}}=100 * \frac{(44.07-39.73)}{44.07}=9.86 \% .
$$

Similarly, the \% savings of other components are calculated and represented in Figure 6 . The overall \% of cost savings of the assembly is estimated using

$$
\% T S=100 * \frac{\left(T C_{E M}-T C_{P M}\right)}{T C_{E M}},
$$

where 










TABLE 20: The value of $N A_{k}$ for various bin numbers from 5 to 8 , linear assembly.

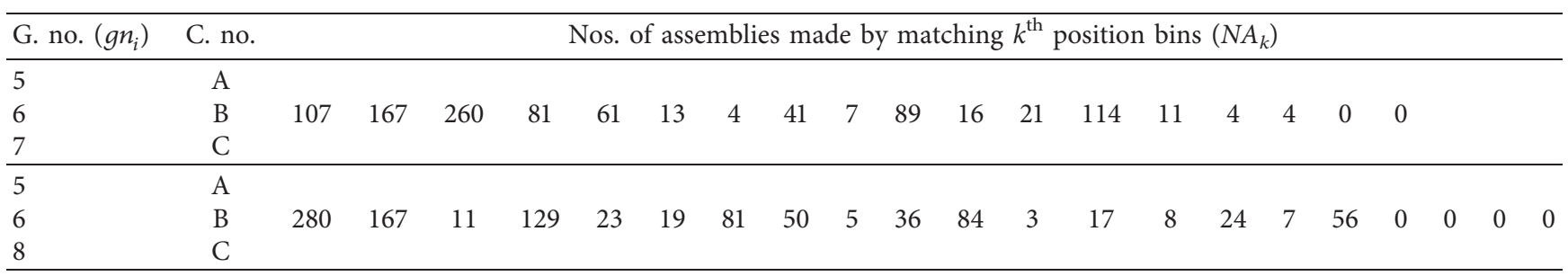

TABLE 21: Details of length of bin for linear assembly.

\begin{tabular}{|c|c|c|c|}
\hline \multirow{2}{*}{ Bin number } & \multicolumn{3}{|c|}{$l b$} \\
\hline & EM & PM & IPM \\
\hline$\left[\begin{array}{lll}5 & 6 & 6\end{array}\right]$ & 16 & 15 & \\
\hline$\left[\begin{array}{lll}4 & 6 & 6\end{array}\right]$ & 16 & 15 & 14 \\
\hline$\left[\begin{array}{lll}4 & 5 & 6\end{array}\right]$ & 16 & 15 & 13 \\
\hline$\left[\begin{array}{lll}5 & 5 & 6\end{array}\right]$ & 16 & 15 & 13 \\
\hline$\left[\begin{array}{lll}4 & 5 & 5\end{array}\right]$ & 13 & 9 & \\
\hline$\left[\begin{array}{lll}5 & 6 & 7\end{array}\right]$ & 19 & 18 & \\
\hline$\left[\begin{array}{lll}5 & 6 & 8\end{array}\right]$ & 22 & 21 & \\
\hline$\left[\begin{array}{lll}6 & 5 & 6\end{array}\right]$ & 16 & 15 & \\
\hline$\left[\begin{array}{lll}6 & 5 & 4\end{array}\right]$ & 16 & 15 & 13 \\
\hline$\left[\begin{array}{lll}5 & 6 & 5\end{array}\right]$ & 16 & 15 & 13 \\
\hline$\left[\begin{array}{lll}5 & 6 & 4\end{array}\right]$ & 16 & 15 & 13 \\
\hline$\left[\begin{array}{lll}3 & 4 & 5\end{array}\right]$ & 13 & 12 & 10 \\
\hline
\end{tabular}

EM: existing method; PM: proposed method; IPM: improvement in proposed method.

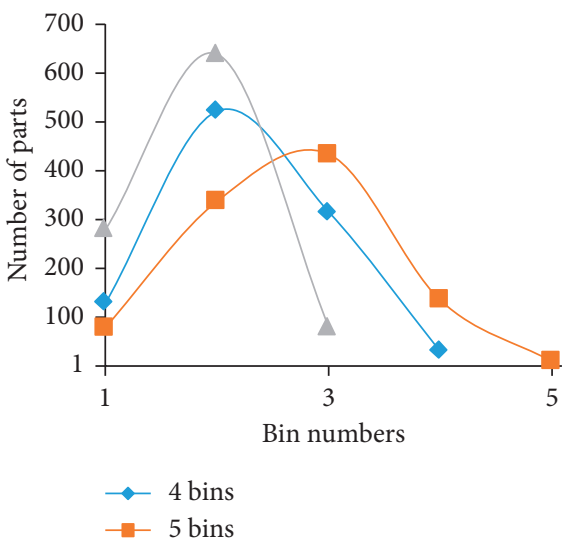

(a)

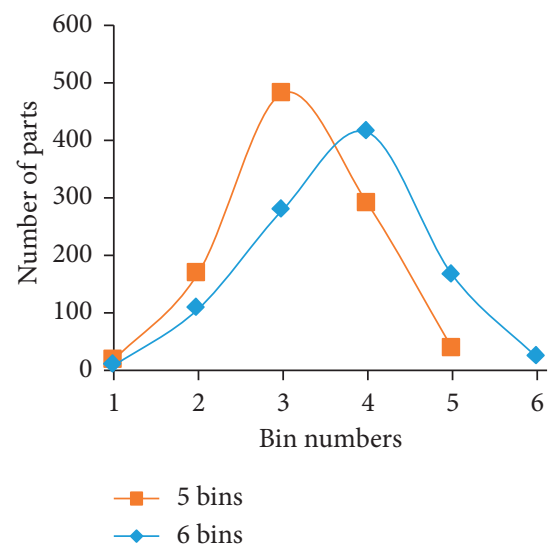

(b)

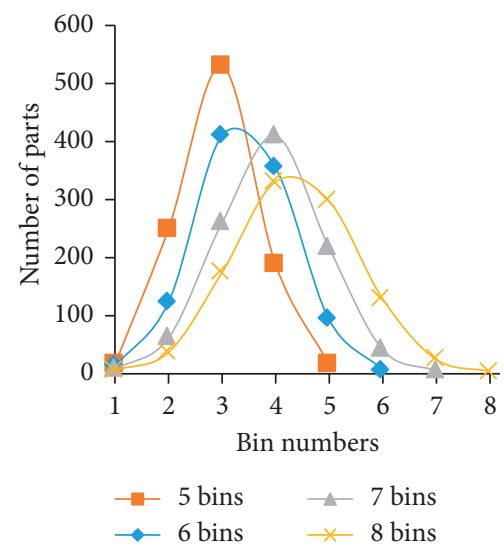

(c)

Figure 4: Dimensional distribution of linear assembly. (a) Gear A. (b) Gear B. (c) Gear C.

$$
\begin{aligned}
T C_{E M} & =\sum_{i=1}^{n c} M C_{i a}=M C_{X_{1} a}+2 M C_{X_{2 a} a}+M C_{X_{2 b} a}+2 M C_{X_{3 a} a}+M C_{X_{3 b} a}+M C_{X_{4} a}+M C_{X_{5} a}, \\
T C_{E M} & =44.07+2 * 139.19+139.19+2 * 143.18+143.18+70.14+47.35=1008.66 . \\
T C_{E M} & =\sum_{i=1}^{n c} M C_{i}=M C_{X_{1}}+2 M C_{X_{2 a}}+M C_{X_{2 b}}+2 M C_{X_{3 a}}+M C_{X_{3 b}}+M C_{X_{4}}+M C_{X_{5}}, \\
T C_{E M} & =39.73+2 * 39.73+39.73+2 * 39.73+39.73+23.92+29.23=331.23, \\
\% T S & =100 * \frac{(1008.66-331.23)}{1008.66}=67.16 \% .
\end{aligned}
$$


TABLe 22: Dimensional distribution of $X_{1}, X_{2 a}, X_{2 b}$, and $X_{3 b}$.

\begin{tabular}{|c|c|c|c|c|c|c|c|c|c|c|c|c|c|c|c|c|}
\hline \multirow{2}{*}{ Bin no. } & \multicolumn{4}{|c|}{$X_{1}$} & \multicolumn{4}{|c|}{$X_{2 a}$} & \multicolumn{4}{|c|}{$X_{2 b}$} & \multicolumn{4}{|c|}{$X_{3 a}$} \\
\hline & 3 & 4 & 5 & 6 & 3 & 4 & 5 & 6 & 3 & 4 & 5 & 6 & 3 & 4 & 5 & 6 \\
\hline 1 & 131 & 46 & 24 & 14 & 191 & 86 & 53 & 35 & 45 & 10 & 5 & 2 & 135 & 49 & 27 & 15 \\
\hline 2 & 687 & 414 & 211 & 117 & 625 & 413 & 256 & 156 & 684 & 253 & 100 & 43 & 724 & 438 & 217 & 120 \\
\hline 3 & 182 & 455 & 448 & 329 & 184 & 413 & 400 & 308 & 271 & 617 & 449 & 218 & 141 & 459 & 478 & 352 \\
\hline 4 & & 85 & 272 & 358 & & 88 & 238 & 317 & & 120 & 395 & 466 & & 54 & 246 & 372 \\
\hline 5 & & & 45 & 152 & & & 53 & 150 & & & 51 & 244 & & & 32 & 126 \\
\hline 6 & & & & 30 & & & & 34 & & & & 27 & & & & 15 \\
\hline
\end{tabular}

Table 23: Dimensional distribution of $X_{3 b}, X_{4}$, and $X_{5}$.

\begin{tabular}{|c|c|c|c|c|c|c|c|c|c|c|c|c|c|}
\hline \multirow{2}{*}{ Bin no. } & \multicolumn{4}{|c|}{$X_{3 b}$} & \multicolumn{4}{|c|}{$X_{4}$} & \multicolumn{5}{|c|}{$X_{5}$} \\
\hline & 3 & 4 & 5 & 6 & 3 & 4 & 5 & 6 & 3 & 4 & 5 & 6 & 7 \\
\hline 1 & 105 & 47 & 26 & 15 & & 79 & 44 & 21 & 58 & 17 & 7 & 5 & 3 \\
\hline 2 & 739 & 425 & 191 & 120 & & 456 & 261 & 148 & 698 & 290 & 120 & 53 & 26 \\
\hline 3 & 156 & 468 & 490 & 352 & & 418 & 463 & 366 & 244 & 601 & 442 & 249 & 143 \\
\hline 4 & & 60 & 263 & 372 & & 47 & 212 & 335 & & 92 & 375 & 449 & 331 \\
\hline 5 & & & 30 & 126 & & & 20 & 118 & & & 56 & 213 & 350 \\
\hline 6 & & & & 15 & & & & 12 & & & & 31 & 125 \\
\hline 7 & & & & & & & & & & & & & 22 \\
\hline
\end{tabular}

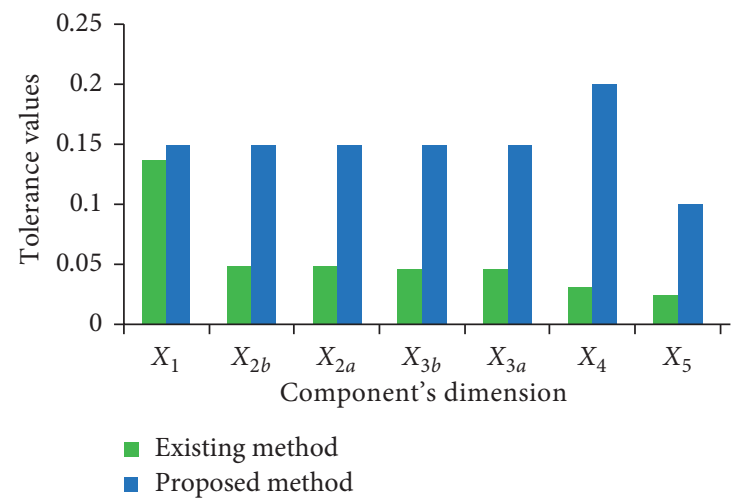

FIGURE 5: Existing and proposed tolerance of component's dimension.

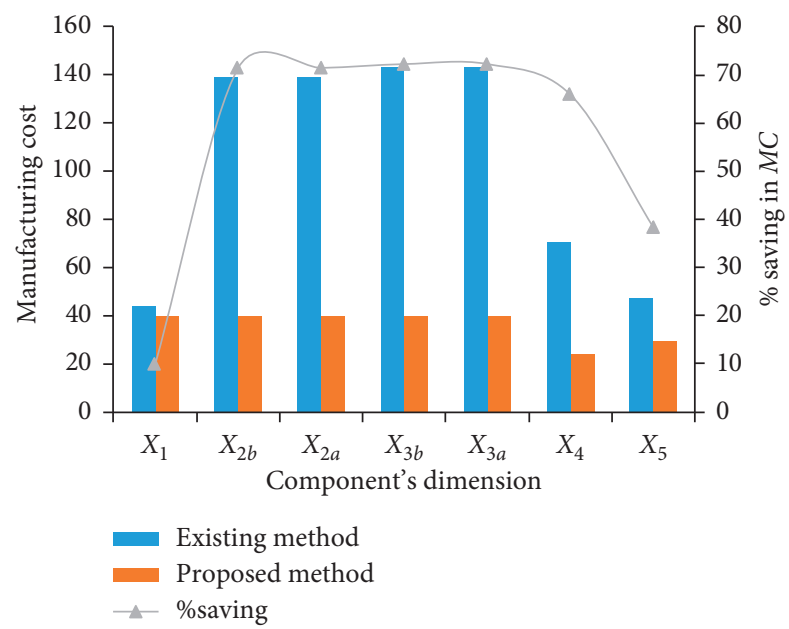

FIgURE 6: Percentage of cost savings in the proposed method. 
TABLE 24: Details of the length of the bin for KJA with three arms.

\begin{tabular}{|c|c|c|c|c|}
\hline Pr. no. & $G$ & EM & $\mathrm{PM}$ & IPM \\
\hline 1 & 1 & 26 & 17 & 16 \\
\hline 2 & 1.5 & 26 & 21 & 16 \\
\hline 3 & 1.5 & 33 & 24 & 23 \\
\hline 4 & 1.8 & 33 & 26 & 22 \\
\hline 5 & 1.5 & 33 & 25 & 24 \\
\hline 6 & 1.5 & 40 & 26 & 23 \\
\hline 7 & 1.5 & 40 & 26 & 25 \\
\hline 8 & 1.5 & 40 & 27 & 26 \\
\hline 9 & 1.5 & 40 & 27 & 26 \\
\hline 10 & 1.5 & 40 & 27 & \\
\hline 11 & 1.8 & 40 & 28 & 27 \\
\hline 12 & 2 & 40 & 29 & \\
\hline 13 & 2 & 47 & 32 & 31 \\
\hline 14 & 2 & 47 & 32 & 31 \\
\hline 15 & 2 & 47 & 32 & 31 \\
\hline
\end{tabular}

Table 11 represents the length of bin $(l b)$, assembly specification, and total assemblies made for various bin numbers of components of KJA with three arms. The best bin combinations which produce the assemblies with the required specification without surplus parts are represented in Tables 12-18. The number of assemblies made in each mating of the $k^{\text {th }}$ position of bin combinations is listed in Table 19. The details of the length of the bin based on the existing and proposed methods are presented in Table 24. Furthermore, the improved length of the bin is also shown.

\section{Conclusion}

In the present work, the interrelated dimensional chain product is manufactured with significantly less manufacturing cost by implementing the novel unequal grouping method. The proposed method is implemented to minimize the manufacturing cost of linear and complex assemblies. In the linear assembly, $14.3 \%$ reduction in clearance variation has been achieved when compared with the the method proposed by Wang et al. [33]. For KJA assembly, as compared to the existing method introduced by Sivakumar et al. [34], the present method can yield $67.16 \%$ of cost savings in manufacturing the assembly without compromising the assembly specification and with nil surplus parts in a single stage. Also, the proposed method reduced the length of bin value by $38.46 \%$ compared with the existing method. From these outcomes, the proposed novel unequal grouping method along with the ABC algorithm is most suitable to solve any linear or complex assembly with $100 \%$ inspection by considering the manufacturing objectives such as the minimization of surplus parts, clearance variation, and manufacturing cost. The work can be extended further to solve the problems associated with nonlinear assemblies using an equal area method or an unequal area method, or an unequal grouping method by considering the manufacturing objectives.

\section{Data Availability}

The data used to support the findings of this study are included within the article.

\section{Conflicts of Interest}

The authors declare no conflicts of interest regarding the publication of this article.

\section{References}

[1] K. C. Chan and R. J. Linn, "A grouping method for selective assembly of parts of dissimilar distributions," Quality Engineering, vol. 11, no. 2, pp. 221-234, 1998.

[2] A. Thesen and A. Jantayavichit, "Design and evaluation of a selective assembly station for high precision scroll compressor shells," vol. 1, pp. 694-700, in Proceedings of the 1999 Winter Simulation Conference "Simulation-A Bridge to the Future" (WSC'99) (Cat. No. 99CH37038), vol. 1, IEEE, Phoenix, AZ, USA, December 1999.

[3] S. Kannan and V. Jayabalan, "A new grouping method to minimize surplus parts in selective assembly for complex assemblies," International Journal of Production Research, vol. 39, no. 9, pp. 1851-1863, 2001.

[4] D. C. Kern, "Forecasting manufacturing variation using historical process capability data: applications for random assembly, selective assembly, and serial processing," Doctoral dissertation, Massachusetts Institute of Technology, Department of Mechanical Engineering, Cambridge, MA, USA, 2003.

[5] S. Matsuura and N. Shinozaki, "Optimal process design in selective assembly when components with smaller variance are manufactured at three shifted means," International Journal of Production Research, vol. 49, no. 3, pp. 869-882, 2011.

[6] G. A. Pugh, "Partitioning for selective assembly," Computers and Industrial Engineering, vol. 11, no. 1-4, pp. 175-179, 1986.

[7] G. A. Pugh, "Selective assembly with components of dissimilar variance," Computers and Industrial Engineering, vol. 23, no. 1-4, pp. 487-491, 1992.

[8] X. D. Fang and Y. Zhang, "Assuring the matchable degree in selective assembly via a predictive model based on set theory and probability method," Journal of Manufacturing Science and Engineering, vol. 118, no. 2, pp. 252-258, 1996.

[9] J. R. Babu and A. Asha, "Tolerance modelling in selective assembly for minimizing linear assembly tolerance variation and assembly cost by using Taguchi and AIS algorithm," 
International Journal of Advanced Manufacturing Technology, vol. 75, no. 5-8, pp. 869-881, 2014.

[10] M. S. Kumar and S. M. Kannan, "Optimum manufacturing tolerance to selective assembly technique for different assembly specifications by using genetic algorithm," International Journal of Advanced Manufacturing Technology, vol. 32, no. 5-6, pp. 591-598, 2007.

[11] N. Manickam and T. N. De, "Optimum group size selection for launch vehicle sections linear assembly by selective assembly method," International Journal of Engineering Research and Technology, vol. 4, pp. 837-843, 2015.

[12] A. R. Aderiani, K. Wärmefjord, and R. Söderberg, "A multistage approach to the selective assembly of components without dimensional distribution assumptions," Journal of Manufacturing Science and Engineering, vol. 140, no. 7, 2018.

[13] Y. Hui, X. Mei, G. Jiang, F. Zhao, Z. Ma, and T. Tao, "Assembly quality evaluation for linear axis of machine tool using data-driven modeling approach," Journal of Intelligent Manufacturing, 2020.

[14] S. G. Ponnambalam, S. S. Sankar, S. Sriram, and M. Gurumarimuthu, "Parallel populations genetic algorithm for minimizing assembly variation in selective assembly," in Proceedings of the 2006 IEEE International Conference on Automation Science and Engineering, pp. 496-500, IEEE, Shanghai, China, October 2006.

[15] T. Malaichamy, R. Sivasubramanian, S. M. Kumar, and M. C. Sundaram, "Simulated annealing algorithm for minimising the surplus parts in selective assembly-a software approach," Asian Journal of Research in Social Sciences and Humanities, vol. 6, no. 9, pp. 1567-1586, 2016.

[16] A. Singh, "An artificial bee colony algorithm for the leafconstrained minimum spanning tree problem," Applied Soft Computing, vol. 9, no. 2, pp. 625-631, 2009.

[17] D. Karaboga and B. Akay, "A modified artificial bee colony (ABC) algorithm for constrained optimization problems," Applied Soft Computing, vol. 11, no. 3, pp. 3021-3031, 2011.

[18] D. Karaboga and C. Ozturk, "A novel clustering approach: artificial bee colony (ABC) algorithm," Applied Soft Computing, vol. 11, no. 1, pp. 652-657, 2011.

[19] L. Asadzadeh, "A parallel artificial bee colony algorithm for the job shop scheduling problem with a dynamic migration strategy," Computers \& Industrial Engineering, vol. 102, pp. 359-367, 2016.

[20] G. Tian, M. Zhou, and P. Li, "Disassembly sequence planning considering fuzzy component quality and varying operational cost," IEEE Transactions on Automation Science and Engineering, vol. 15, no. 2, pp. 748-760, 2017.

[21] G. Tian, Y. Ren, Y. Feng, M. Zhou, H. Zhang, and J. Tan, "Modeling and planning for dual-objective selective disassembly using AND/OR graph and discrete artificial bee colony," IEEE Transactions on Industrial Informatics, vol. 15, no. 4, pp. 2456-2468, 2018.

[22] X. Li, Y. Huang, Y. Zhou, and X. Zhu, "Robot path planning using improved artificial bee colony algorithm," in Proceedings of the 2018 IEEE 3rd Advanced Information Technology, Electronic and Automation Control Conference (IAEAC), pp. 603-607, IEEE, Chongqing, China, October 2018.

[23] M. Chen, "Improved artificial bee colony algorithm based on escaped foraging strategy," Journal of the Chinese Institute of Engineers, vol. 42, no. 6, pp. 516-524, 2019.

[24] X. Xu, J. Hao, and Y. Zheng, "Multi-objective artificial bee colony algorithm for multi-stage resource leveling problem in sharing logistics network," Computers \& Industrial Engineering, vol. 142, Article ID 106338, 2020.
[25] C. He, Y. Zhang, and D. Gong, "A pseudo-label guided artificial bee colony algorithm for hyperspectral band selection," Remote Sensing, vol. 12, no. 20, p. 3456, 2020.

[26] X.-H. Wang, Y. Zhang, X.-Y. Sun, Y.-L. Wang, and C.-H. Du, "Multi-objective feature selection based on artificial bee colony: an acceleration approach with variable sample size," Applied Soft Computing, vol. 88, Article ID 106041, 2020.

[27] Z. Yong, H. Chun-lin, S. Xian-fang, and S. Xiao-yan, "A multistrategy integrated multi-objective artificial bee colony for unsupervised band selection of hyperspectral images," Swarm and Evolutionary Computation, vol. 60, Article ID 100806, 2021.

[28] H. Gao, Z. Fu, C. M. Pun, J. Zhang, and S. Kwong, “An efficient artificial bee colony algorithm with an improved linkage identification method," IEEE Transactions on $C y$ bernetics, 2020.

[29] L. Le Dinh, D. Vo Ngoc, and P. Vasant, "Artificial bee colony algorithm for solving optimal power flow problem," The Scientific World Journal, vol. 2013, Article ID 159040, 9 pages, 2013.

[30] D. Karaboga and B. Basturk, "Artificial bee colony (ABC) optimization algorithm for solving constrained optimization problems," in International Fuzzy Systems Association World Congress, pp. 789-798, Springer, Berlin, Germany, 2007.

[31] D. Karaboga and B. Basturk, "On the performance of artificial bee colony (ABC) algorithm," Applied Soft Computing, vol. 8, no. 1, pp. 687-697, 2008.

[32] L. Nagarajan, S. K. Mahalingam, S. Gurusamy, and V. K. Dharmaraj, "Solution for bi-objective single row facility layout problem using artificial bee colony algorithm," European Journal of Industrial Engineering, vol. 12, no. 2, pp. 252-275, 2018.

[33] W. Wang, D. Li, and J. Chen, "Minimizing assembly variation in selective assembly for complex assemblies using genetic algorithm," in Proceedings of the 2011 Second International Conference on Mechanic Automation and Control Engineering, pp. 1401-1406, IEEE, Inner Mongolia, China, July 2011.

[34] K. Sivakumar, C. Balamurugan, and S. Ramabalan, "Simultaneous optimal selection of design and manufacturing tolerances with alternative manufacturing process selection," Computer-Aided Design, vol. 43, no. 2, pp. 207-218, 2011. 